\title{
Multiple Dehydrogenation Reactions of Negative Ions in Low Pressure Silane Plasma Chemistry
}

\author{
A A Howling ${ }^{1}$, A Descoeudres ${ }^{2}$, Ch. Hollenstein ${ }^{1} \ddagger$ \\ ${ }^{1}$ Ecole Polytechnique Fédérale de Lausanne (EPFL), Swiss Plasma Center (SPC), \\ CH-1015 Lausanne, Switzerland \\ 2 Swiss Center for Electronics and Microtechnology (CSEM), PV-Center, \\ Jaquet-Droz 1, 2002 Neuchâtel, Switzerland \\ E-mail: alan.howling@epfl.ch
}

\begin{abstract}
Micro-particle formation in low pressure silane $\left(\mathrm{SiH}_{4}\right)$ plasmas has been of technical interest and concern for at least 40 years. Negative ion plasma chemistry is a candidate for the initial nucleation, which has been extensively studied both experimentally and theoretically. Nevertheless, the nature and mechanisms of anionneutral reactions remain uncertain in models of nucleation.

In the pure silane, 0.1 mbar plasma of this work, silicon hydride anion mass spectra are interpreted in terms of iterative anion-silane reactions, involving release of one, or two, hydrogen molecules. Such "multiple dehydrogenation" has been directly observed by Operti, Rabezzana and Vaglio [Rapid Commun. Mass Spectrom. 20, 2696 (2006)], where triple dehydrogenation also occurred.

A statistical model, defined uniquely by a branching ratio of $68 \%$ for single hydrogenation, and $32 \%$ for double dehydrogenation, gives an accurate fit to the hydrogen distribution for all heavy silicon hydride anions (abbreviated here by "silanions"), up to at least 800 atomic mass units.

Most theoretical models of silanion-neutral nucleation consider dehydrogenation where no more than one $\mathrm{H}_{2}$ molecule is released. It is suggested that multiple dehydrogenation could improve the comparison of theory with experiments.
\end{abstract}

$\ddagger$ Retired. Present address: Av. William 46, CH-1095 Lutry, Switzerland 


\section{Introduction}

Negatively-charged silicon hydride molecules are of industrial interest regarding microparticle formation in silane $\left(\mathrm{SiH}_{4}\right)$ plasma-enhanced deposition of silicon [1] for large-area electronics (thin-film photovoltaic solar cells, thin-film transistors for flat displays, etc), as well as in nano-particle synthesis [2], astrophysics and fundamental studies. These anionic clusters, $\mathrm{Si}_{n} \mathrm{H}_{m}^{-}$, are confined inside $\mathrm{RF}$ plasmas by the sheath potentials, and hence have the possibility to become the pathway for polymerisation over many seconds during high collision rates at plasma processing pressures $(\sim 0.1$ mbar, for example) [3]. The growth mechanisms, and the effect on the plasma of negative ions [4] and particles, have been extensively modelled by many authors $[1,5,6,7,8,9,10,11,12,13,14,15,16,17,18,19,20,21,22,23,24]$, and continue to be a topical subject. Indeed, low-pressure silane-containing plasmas in which silicon nanoparticles nucleate and grow are probably the most studied, both experimentally and numerically, in terms of particle formation in non-thermal plasmas [1].

However, the experimental measurements of $\mathrm{Si}_{n} \mathrm{H}_{m}^{-}$in plasma processing conditions were reported by only two groups in the 1980's [5] and 1990's [25, 26, 27, 28, 29, 30, 31, 32,33 , and many questions still remain before uncertainties can be removed for reliable modeling [1]. Fortunately, fundamental studies of negative gas-phase ion chemistry in silane have subsequently been performed independently by other groups, using very different techniques $[34,35,36]$. These important results are reviewed and applied to silicon hydride anion measurements in the present work.

We propose the term "silanion" $\S[29,35]$ as a shorthand contraction of "silane" and "anion" to mean a silicon hydride negative ion, $\mathrm{Si}_{n} \mathrm{H}_{m}^{-}$. This is in a similar sense to the 1933 proposition of "carbanion" as a negatively-charged carbon molecular ion [38]. For the silanions $\mathrm{Si}_{n} \mathrm{H}_{m}^{-}$, we use $n$ for the number of $\mathrm{Si}$ atoms, and $m$ for the number of $\mathrm{H}$ atoms, throughout.

In this work, we first describe the experimental conditions used to measure the silanion mass spectrum in Sec. 2, with a consideration of the silanion dynamics in the optimized time intervals of plasma power modulation. Other more recent techniques for measuring silanions are reviewed in Sec. 3. The extraction dynamics of silanions are compared, in Sec. 4, with three other electronegative gas plasmas to confirm that the silanions do originate within the plasma period, and not from the afterglow, nor from surface reactions.

In Sec. 5, the measured mass spectrum of small silanions $(n \leq 6)$ is deconvolved by the silicon isotope distribution to identify the main dehydrogenation reactions and to compare with experiments and ab initio calculations in the literature. Conversely, in Sec. 6 , the heavier silanions are first modeled by a binomial distribution of dehydrogenation reactions, before convolution with the silicon isotopes, in order to compare with the measured mass spectrum in Sec. 7. The principal result in the Conclusions is that multiple dehydrogenation reactions, as directly observed by Operti et al [35], can $\S$ No relation to "Silanion", a Greek sculptor in the fourth century BC [37]. 
accurately account for the measured mass spectrum up to at least $800 \mathrm{amu}$. This suggests that silanion-neutral nucleation models could be improved by including multiple deydrogenation reactions.

\section{Experimental description and the measured mass spectrum}

\subsection{Reactor set-up and the mass spectrometer}

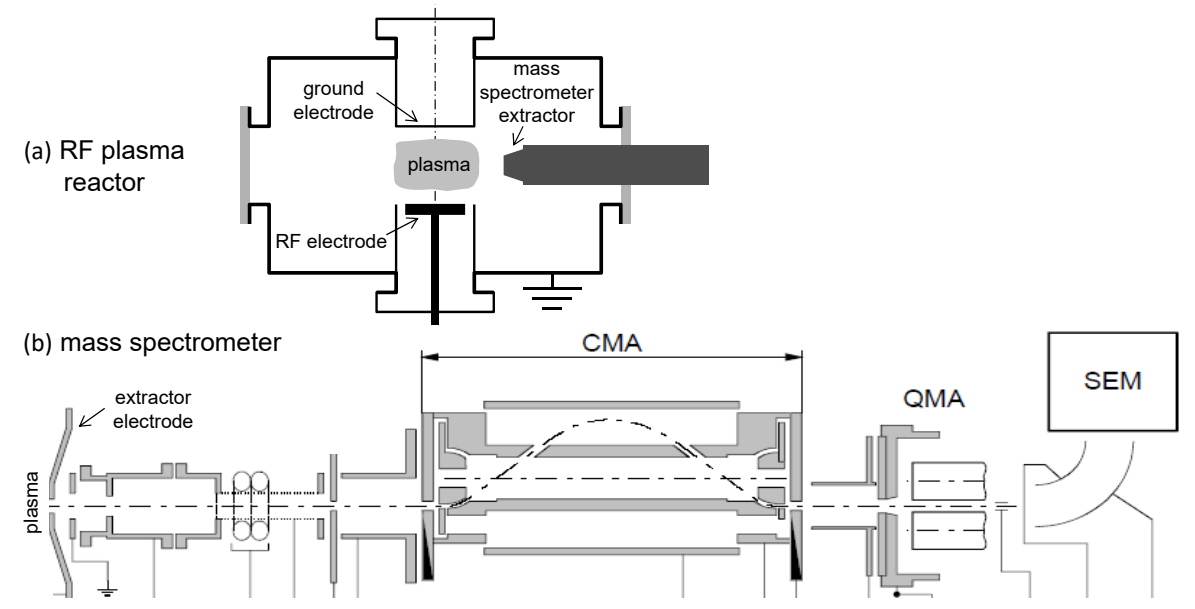

Figure 1. (a) RF plasma reactor (not to scale) with cylindrical electrodes $13 \mathrm{~cm}$ diameter, $2.5 \mathrm{~cm}$ apart, showing the mass spectrometer to the side. (b) Schematic of the Plasma Process Monitor PPM421 spectrometer, showing the extractor electrode $(+5 \mathrm{~V})$ with a $0.1 \mathrm{~mm}$ diameter orifice, the cylindrical mirror energy analyser (CMA), the quadrupole mass analyser (QMA cut view), and the secondary electron multiplier (SEM) detector.

The experimental apparatus shown in Fig. 1 is a parallel plate capacitively-coupled reactor comprising two stainless steel cylindrical electrodes $13 \mathrm{~cm}$ in diameter with a $2.5 \mathrm{~cm}$ electrode gap; the ground electrode was heated to $200{ }^{\circ} \mathrm{C}$ (for more details, see [27]). The plasma parameters used here were pure silane at pressure 0.1 mbar with 30 sccm flow, and RF power $\left(\sim 0.03 \mathrm{~W} \mathrm{~cm}^{-3}\right)$ at an excitation frequency of $13.56 \mathrm{MHz}$, which was $\mathrm{ON} / \mathrm{OFF}$ modulated at $\mathrm{kHz}$ frequency (see Sec. 2.3) to enable measurements of the negative ions during the afterglow [27].

A Pfeiffer Vacuum Plasma Process Monitor type PPM421, comprising a doublefocussing cylindrical mirror energy analyser (CMA) [39] and quadrupole mass analyser (QMA), was used to detect neutral radicals and ions (positive and negative) with a mass range up to 2000 amu. Time-resolved ion flux measurements were made using an EG\&G Ortec multichannel scalar. The probe head, with $0.1 \mathrm{~mm}$ diameter extractor orifice, was positioned adjacent to the electrode gap at approximately $10 \mathrm{~mm}$ from the plasma visual boundary with a $+5 \mathrm{~V}$ bias on the extractor electrode facing the plasma (Fig. 1). Energy-resolved measurements were not required in this work, so the anion transmission energy was set with low resolution at $\sim 3 \pm 1 \mathrm{eV}$. The question of non- 
perturbative anionic cluster sampling was addressed in Ref. [29]. Negative ions are trapped in continuous plasmas by the sheath potential drop at the walls. In this work, $\mathrm{ON} / \mathrm{OFF}$ power pulsing was used to collapse the sheaths so that negative ions were released to drift and diffuse to the plasma monitor, as described in Sec. 2.3 and Sec. 4 .

Experimental difficulties include spurious charging of films deposited on electrode surfaces and eventual clogging of the extractor $0.1 \mathrm{~mm}$ diameter pinhole [40]. This necessitated demounting of the spectrometer head for cleaning in acid after a few tens of minutes of exposure to silane plasma, which could explain the limited number of groups reporting results in silane plasmas.

\subsection{Measured mass spectrum}
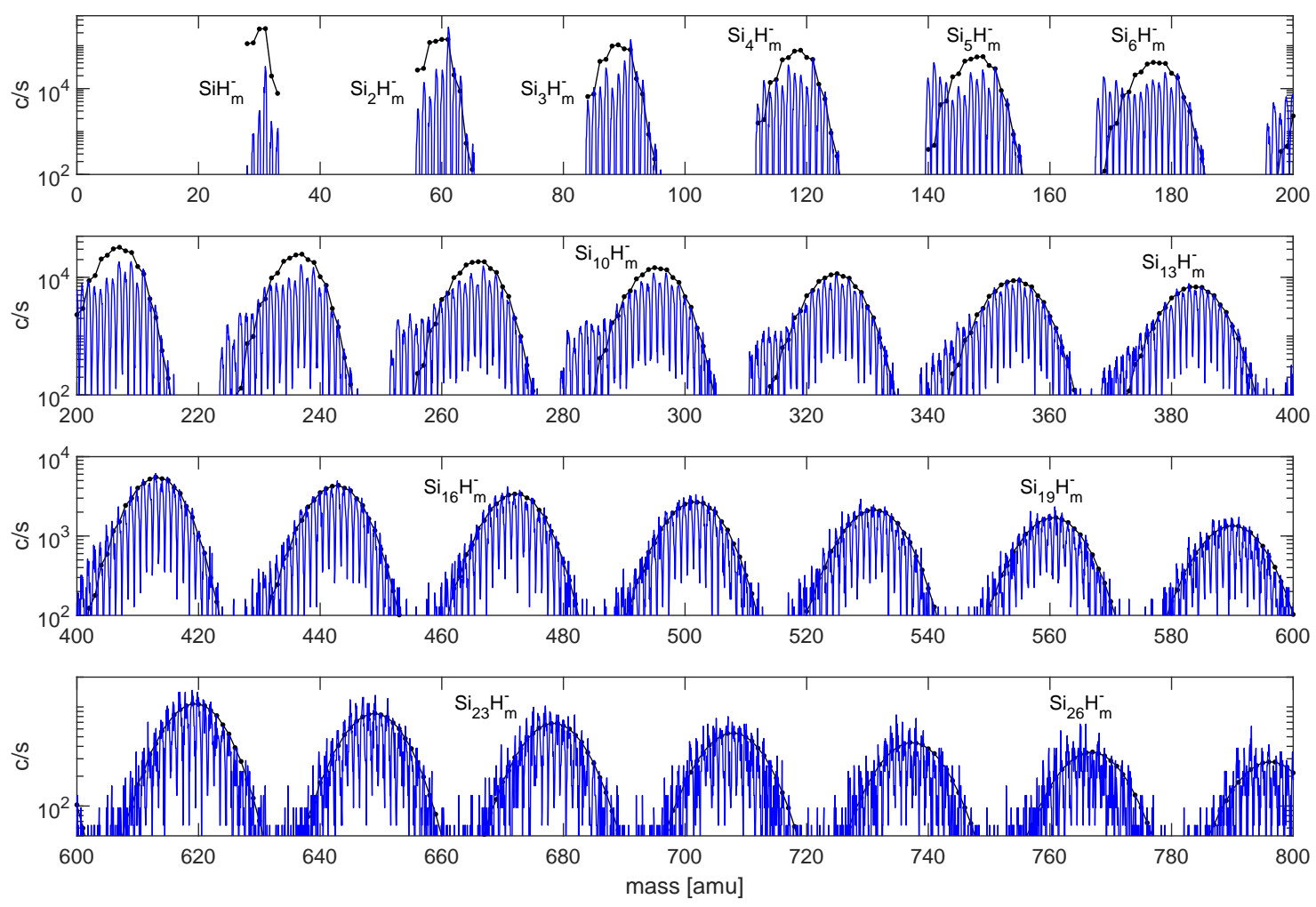

Figure 2. Mass spectrum raw data, for 1 to 800 atomic mass units (amu), of silanions from a modulated RF plasma with $30 \mathrm{sccm}$ flow of pure silane at 0.1 mbar pressure. The RF power $\left(0.03 \mathrm{~W} \mathrm{~cm}^{-3}\right)$, at an excitation frequency of $13.56 \mathrm{MHz}$, was $\mathrm{ON} / \mathrm{OFF}$ modulated with $t_{\mathrm{on}}=110 \mu \mathrm{s}$ and $t_{\mathrm{off}}=300 \mu \mathrm{s}$. The ordinate units are in counts per second. The mass range 0 - $200 \mathrm{amu}$ is shown in more detail in Fig. 9, and the group $\mathrm{Si}_{19} \mathrm{H}_{m}^{-}$is detailed in Fig. 13. The points joined by the black line represent the model mass spectrum calculated in Sec. 6 .

Fig. 2 shows a single mass spectrum acquisition up to $800 \mathrm{amu}$, with mass resolution better than 1 amu over the whole mass range, 16 datapoints per amu, and a signal dynamic range from 32 counts per second $(\mathrm{c} / \mathrm{s})$ up to $2.7 \times 10^{5} \mathrm{c} / \mathrm{s}$. Using these plasma parameters, the power modulation times $t_{\mathrm{on}}=110 \mu \mathrm{s}$ and $t_{\mathrm{off}}=300 \mu \mathrm{s}$, were empirically 
"tuned" for optimal anion generation, de-trapping, and drift/diffusion, to obtain the maximum anion signal and mass for this particular set-up, as described in Sec. 2.3. The spectrum is rich in silanions, with every intermediate mass represented in all clusters: There are no forbidden masses, so every corresponding neutral must have an electron affinity greater than zero. The continuous black lines in the figure represent a reaction sequence model which is explained in Sec. 6 .

As a preliminary analysis of this mass spectrum, Fig. 3 shows the mass of the peak of each $\mathrm{Si}_{n} \mathrm{H}_{m}^{-}$anion cluster as a function of its number of Si atoms, $n$, starting from $n=7$. The result is a remarkably straight line through the origin of gradient $29.472 \pm 0.01 \mathrm{amu}$. Stable silicon isotopes consist of masses $28 \mathrm{amu}$ (fractional abundance 0.9221), $29 \mathrm{amu}$ (0.0470), and $30 \mathrm{amu}$ (0.0309), having an average mass 28.109 amu. We deduce that the peak clusters contain $1.363( \pm 0.01) n \mathrm{H}$ atoms, i.e. 1.363 $\mathrm{H}$ atoms per Si atom for every cluster that has a well-defined maximum $(n \geq 7) \|$. These statistics are used to construct a reaction sequence model in Sec. 6, where the constancy of the gradient is tentatively explained in terms of localized growth reactions in Sec. 7.1.

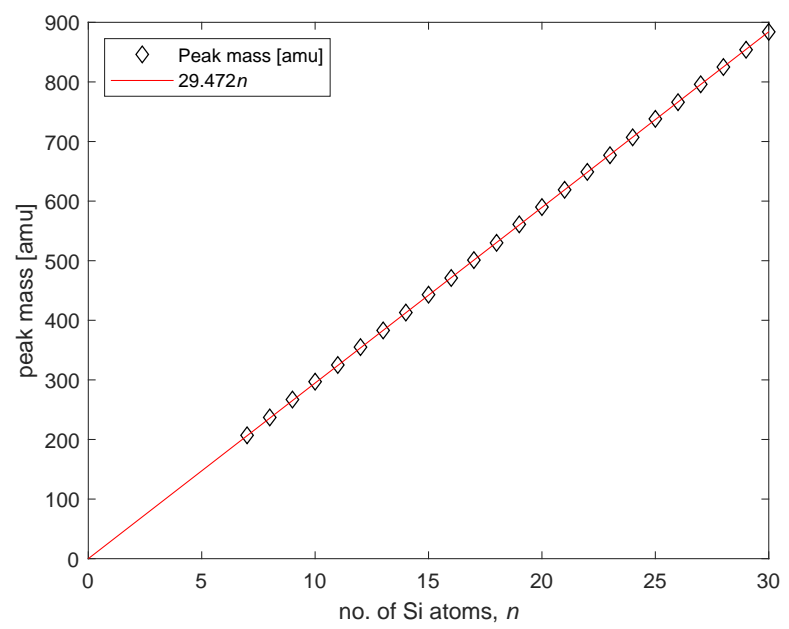

Figure 3. Straight line fit to the mass values (amu) of the peak abundances for each of the silanions $\mathrm{Si}_{n} \mathrm{H}_{m}^{-}$, as a function of the number of silicon atoms for $n \geq 7$.

\subsection{Time-resolved fluxes of silanions}

The power ON/OFF modulation times $t_{\mathrm{on}}=110 \mu \mathrm{s}$ and $t_{\mathrm{off}}=300 \mu \mathrm{s}$ were empirically chosen to give the experimentally-observed highest mass range and count-rates for the silanion mass spectrum. A reason for these time values can be found in the timeresolved fluxes shown in Fig. 4, where the mass resolution was degraded to obtain higher count-rates for each $n$th $\mathrm{Si}_{n} \mathrm{H}_{m}^{-}$group. The time axis of each flux was corrected

$\|$ The difference between the exact mass and the nearest integer mass is $\sim-0.023 \mathrm{amu}$ for silicon isotopes, and $\sim+0.008$ amu for hydrogen isotopes. For a silanion of type $\mathrm{Si}_{n} \mathrm{H}_{1.363 n}^{-}$, the combined difference is $\sim-0.01 n$ amu. Hence for $n \leq 27$ in Fig. 2, this mass difference is $\leq-0.3 \mathrm{amu}$ in a total mass of $800 \mathrm{amu}$. Therefore, integer masses are used in this work. 

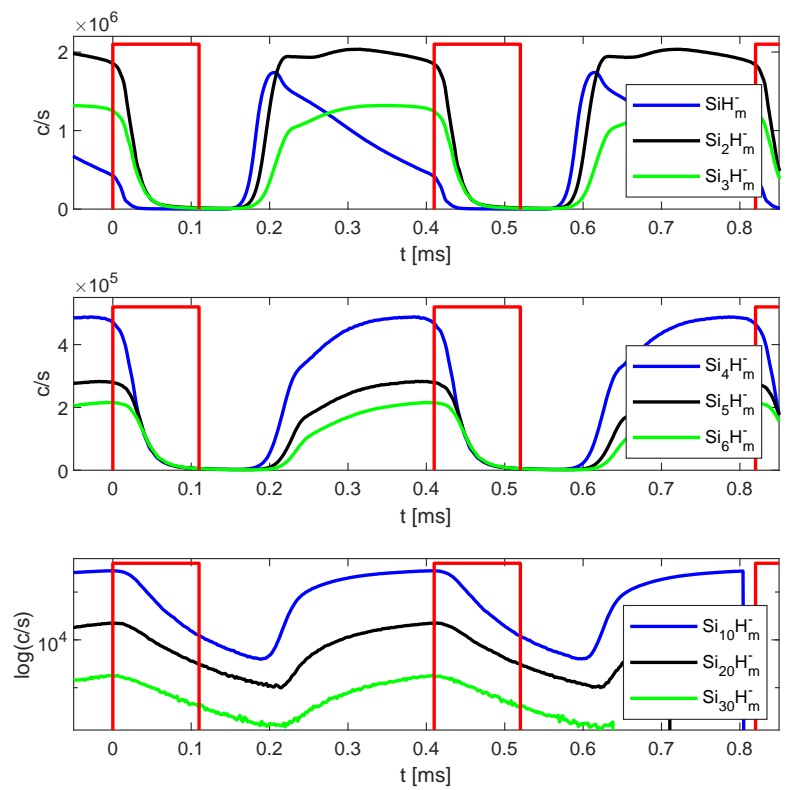

Figure 4. Negative ion fluxes as a function of time in silane plasma for the same conditions as Fig. $2\left(t_{\mathrm{on}}=110 \mu \mathrm{s}\right.$ and $\left.t_{\mathrm{off}}=300 \mu \mathrm{s}\right)$. Top: Mono-, di-, and trisilanions ( $n=1,2,3$ silicon atoms); middle: $n=4,5,6$; bottom: $n=10,20,30$. The top two graphs have a linear scale for the counts per second $(\mathrm{c} / \mathrm{s})$, the bottom scale is logarithmic. Red lines mark the plasma ON period. The time axis is corrected for time-of-flight through the mass spectrometer.

for its transit time through the spectrometer by synchronising the abrupt fall-off of each flux with the onset of the plasma. When the RF power was switched off, following a delay for the sheath to collapse, the silanion fluxes began to be observed (this delay is discussed further in Sec. 4). The mono-silanion group $\mathrm{SiH}_{m}^{-}$rises steeply and then decays during the afterglow before the next power ON period, when the remainder are trapped again within the plasma by the sheath potential. The di-silanions $\mathrm{Si}_{2} \mathrm{H}_{m}^{-}$ rapidly reach a plateau before being trapped. The tri-silanions $\mathrm{Si}_{3} \mathrm{H}_{m}^{-}$drift/diffuse more slowly but still reach a plateau during the afterglow. In successively heavier groups, $n=4,5,6,10,20,30$, the signals rise more and more slowly, without reaching a plateau. Then the empirical optimisation of the $\mathrm{ON}$ and $\mathrm{OFF}$ periods can be qualitatively explained as follows:

(i) The afterglow of $300 \mu \mathrm{s}$ is long enough for the heavy silanions to drift/diffuse to the spectrometer after the sheath collapse delay, but not so long that the smallest anions are completely emptied.

(ii) The plasma duration of $110 \mu$ s is sufficiently long to replenish the anions lost during the afterglow, but not so long that silicon particles begin to form and accumulate [31].

(iii) The overall period of $410 \mu$ s is short enough to give a high repetition frequency of $2.44 \mathrm{kHz}$, providing a high average count rate. 


\section{Review of techniques for silanion measurements}

Howling and Hollenstein et al used two types of energy-resolved quadrupole mass spectrometers to measure silanions during the 1990's: A Hiden Analytical Limited Plasma Monitor HAL-EQP500 45-degree-sector electrostatic energy analyser [25, 26, 27, 28, 29, 30, 31, 33], and a Pfeiffer Vacuum, Balzers Plasma Process Monitor PPM421 described above [29, 31, 32, 33]. The technique of power-modulated plasma and positive extractor voltage was used in both cases $\boldsymbol{q}$. Hence, it is crucial to compare these silanion measurements with other independent sources. In this section, we compile results from subsequent experiments during the last 20 years.

In this context, Negative gas-phase ion chemistry of silane: a quadrupole ion trap study by Operti et al in 2006 is of capital importance [35]. The results were obtained using a very different method of ion trap mass spectrometry (ITMS), which does not involve extraction from a power-modulated plasma. Silanion-neutral chemical ionisation reactions readily occurred at a relatively high pressure for a resonant ion trap, $\sim 1.2 \times 10^{-4}$ mbar, although still only $0.12 \%$ of the silane pressure in the present work. Furthermore, the ion trap can isolate individual silanions to measure their reactions with silane, in contrast to the overall cumulative mass spectrum observed in the present pulsed-plasma experiments. Since the ionisation event may provide the reactant silanion with excess energy, helium buffer gas was used with the aim of quenching excited states to investigate intrinsic (ground state) reactivity with silane [41]. It was observed that the most frequent silanion-silane condensation steps are followed by single and double dehydrogenation; even triple dehydrogenation was observed. Further reference to these results is made from Sec. 5 onwards. Such quantitative data are necessary to remove the considerable uncertainties for silanion nucleation modelling [1].

Similarly to silane plasmas, germane $\left(\mathrm{GeH}_{4}\right)$ plasma negative ions are also quite efficient in the formation and growth of clusters by extensive anion-neutral condensation reactions involving multiple dehydrogenation [35, 42]. The loss of two $\mathrm{H}_{2}$ molecules is easier for anion reactions than for positive ions. Large anionic clusters are formed as the pressure increases using a triple quadrupole mass spectrometer from 25 to 450 mTorr [43]. Negative ions in fact display a higher number of reaction pathways than the corresponding positive ion species [42]. Note also that silanions react efficiently with diborane, more so than positive ions, whereas diborane anions are unreactive toward silane in ITMS experiments [44].

I A remaining question is the apparent attachment to neutral clusters immediately after power off, especially for lower-mass clusters, observed only by the Hiden mass spectrometer [27]. This could be for three possible reasons: 1) The Hiden measurements were made directly across the RF sheath, whereas the Balzers measurements were made off to the side of the plasma; 2) the Hiden high-voltage $(+50 \mathrm{~V})$ extractor electrode was situated behind a $5 \mathrm{~mm}$ diameter grounded orifice, with a $1 \mathrm{~mm}$ gap between them, whereas the Balzers low-voltage $(+5 \mathrm{~V})$ extractor electrode was directly exposed to the plasma; and 3) The Hiden time-resolved signals were measured step-by-step on an oscilloscope, whereas the Balzers spectrometer was connected to a multichannel scalar. Furthermore, the pulse period and duty factor were different. 
Haaland [45] employed Fourier transform ion cyclotron resonance (FTICR) mass spectrometry to measure dissociative electron attachment of silane. This ultra-low pressure $\left(<10^{-6}\right.$ mbar $)$ technique monitors single collision events, which represent the lowest limit for possible anion reaction types and rates [29]. Reents [34] adapted FTICR mass spectrometry for higher pressures up to $5 \times 10^{-4}$ torr by using gas pulsing, and found that negative ion formation efficiency in silane was greatly increased. In general, the production of negative ions is greatly enhanced at higher gas pressures [35, 46], possibly because the exothermicity of the reactions has to be removed by collisions before the product anion redissociates [47]. Note also that silanions are electrostatically trapped in plasmas which greatly increases their reaction time compared to neutrals and positive ions $[3,5,10,27]$.

Peppernick et al [36] produced heavy silanions $\mathrm{Si}_{n} \mathrm{H}_{m}^{-}, n=8-12,0 \leq m \leq 25$ using pulsed silane gas flow over a laser-induced transition metal plasma; the silanions were detected by time-of-flight mass spectrometry up to mass $361 \mathrm{amu}$. This technique, again very different from pulsed RF plasmas, clearly shows series of silanion dehydrogenation reactions which contribute to nucleation processes and micro-particle formation.

Mizutani et al [48] extracted negative ions from a continuous etching plasma by combining a high voltage $(80 \mathrm{~V})$ extractor electrode and electrostatic lens with a permanent magnet filter. Finally, pure silicon clusters formed by magnetron sputtering formed more anions than cations, and the range from $\mathrm{Si}_{4}^{-}$up to $\mathrm{Si}_{1000}^{-}$were measured using UV photoelectron spectroscopy in a time-of-flight mass spectrometer [49].

Some of these experimental results are compared with the mass spectrum analysis in Sec. 5, along with $a b$ initio calculations from the literature.

\section{Extraction dynamics and the origin of anions}

Because pulsed-power plasmas are used here to measure anions, it is crucial to understand the origin of the silanions in Fig. 2 and how they are formed. For example, they cannot be implicated in silanion-neutral nucleation and particle formation if they are artefacts of the diagnostic technique. Specifically, is it correct to assume that the measured silanions are representative of anions accumulated in a continuous silane plasma, or, on the other hand, are they created by attachment in the afterglow, or by surface interactions?

Using the same experimental set-up, the dynamics of pulsed plasmas in three other electronegative gases were used to provide characteristic examples of the three anion origins above, namely [50, 51]:

(i) $\mathrm{CF}_{4}$ plasma in Fig. 5(a): Volume production of $\mathrm{CF}_{x}^{-}$and $\mathrm{F}^{-}$anions [52] occurs by resonant dissociative attachment by high energy electrons during the plasma. Following sheath collapse after a delay of $0.12 \mathrm{~ms}$ in the afterglow [53, 54], these anions are lost by drift-diffusion to electrode surfaces and the extractor electrode.

(ii) $\mathrm{SF}_{6}$ plasma in Fig. 5(b): Cold electrons, which are formed instantly in the afterglow, attach very efficiently to $\mathrm{SF}_{6}$ to form $\mathrm{SF}_{6}^{-}$by $s$-capture [55]. The sheath collapses 

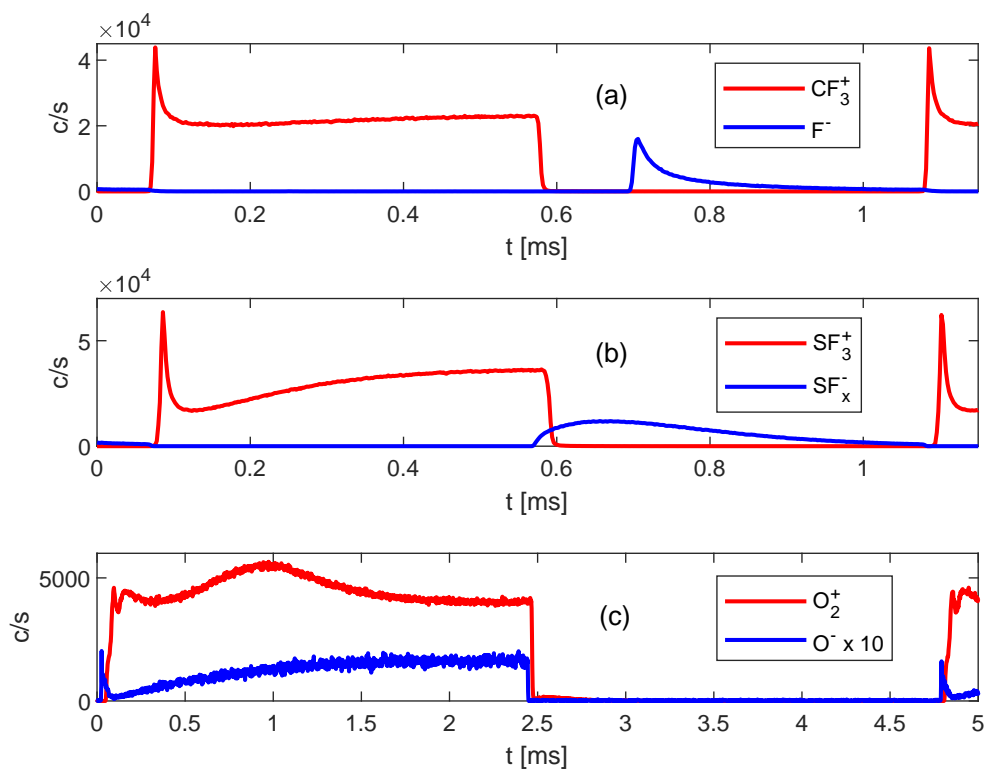

Figure 5. Positive and negative ion fluxes as a function of time in a) $\mathrm{CF}_{4}$, b) $\mathrm{SF}_{6}$, c) $\mathrm{O}_{2}$, in square-wave modulated plasmas

immediately (much sooner than $0.12 \mathrm{~ms}$ ), therefore anions can reach the walls and the extractor electrode at the very beginning of the afterglow.

(iii) $\mathrm{O}_{2}$ plasma in Fig. 5(c): A small flux of negative ions is measured during the discharge, but no negative ions are observed during the afterglow because detachment reactions of $\mathrm{O}^{-}$by $\mathrm{O}_{2}\left(\mathrm{a}^{1} \Delta_{g}\right)$ metastables are very effective. The negative ions observed during the discharge must result from indirect formation at surfaces and the mass spectrometer orifice [50, 56].

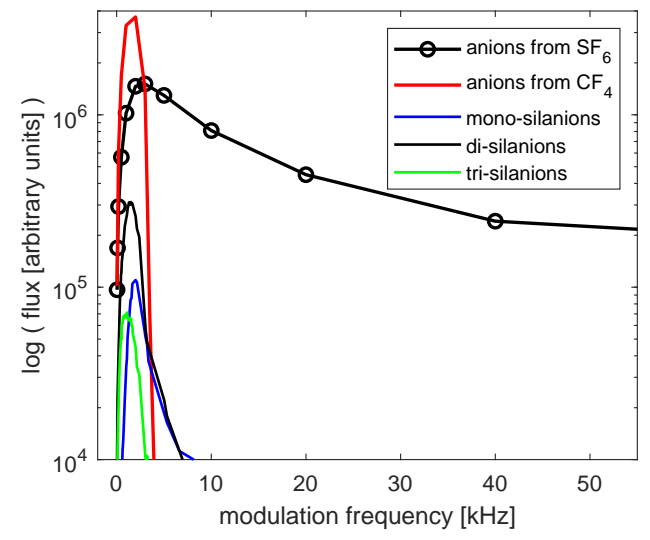

Figure 6. Negative ion fluxes from square-wave $\mathrm{ON} / \mathrm{OFF}$ modulated plasmas in $\mathrm{SF}_{6}$, $\mathrm{CF}_{4}[50]$ and $\mathrm{SiH}_{4}[25]$ (arbitrary intensities, log scale).

The time delay for sheath collapse explains the different dependence of the negative ion fluxes with the modulation frequency in Fig. 6. The delay for $\mathrm{CF}_{4}$ plasma results in a cut-off frequency at $4 \mathrm{kHz}$. At higher frequencies, the modulation period is shorter than 
the sheath collapse time, and therefore negative ions can no longer escape the plasma. In $\mathrm{SF}_{6}$ plasma, the sheath collapse is so fast that negative ions can be measured up to $100 \mathrm{kHz}$. The similarities between $\mathrm{CF}_{4}$ and $\mathrm{SiH}_{4}$ plasmas in Figs. 4, 5 and 6 show, in conclusion, that silanions are formed in the plasma, as in $\mathrm{CF}_{4}$ plasma, and not in the afterglow. Therefore, silanions are indeed candidates for anion-neutral nucleation in particle formation of silane plasmas.

These three characteristic behaviours can be explained by reference to their different electron attachment cross-sections, shown in Fig. 7, along with the ionisation crosssections which are quite similar for the three gases $[50,51,57,58,59,60]$ and silane $[61,62,63] . \mathrm{SF}_{6}$ differs from $\mathrm{O}_{2}, \mathrm{CF}_{4}$ and $\mathrm{SiH}_{4}$ by its much larger attachment crosssection, in particular for very low energy electrons which are dominant by far in the plasma. Negative ions in $\mathrm{CF}_{4}$ and $\mathrm{SiH}_{4}$ are formed only by resonant dissociative electron attachment at electron energies only slightly less than for their ionisation. Just as ionisation occurs only during the plasma, and not during the afterglow, then silanion formation can only occur during the plasma, and not by attachment by cold electrons characteristic of the afterglow $[20,64]$. Attachment to polysilanes was also observed to be by resonant dissociative electron attachment [33]. Note that electron affinity increases with the size of silicon clusters as they tend towards the affinity of bulk silicon [7, 21].

Notwithstanding other experimental demonstrations of silanion reactions [35, 36], it would be instructive to measure silanions during continuous plasma by direct observation. For example, in situ spectroscopic identification of negative ions could be used, such as cavity ring-down spectroscopy of $\mathrm{F}^{-}$in $\mathrm{CF}_{4}$ plasma [52], although absorption methods can be hampered by light scattering from particles formed in silane plasmas [65].

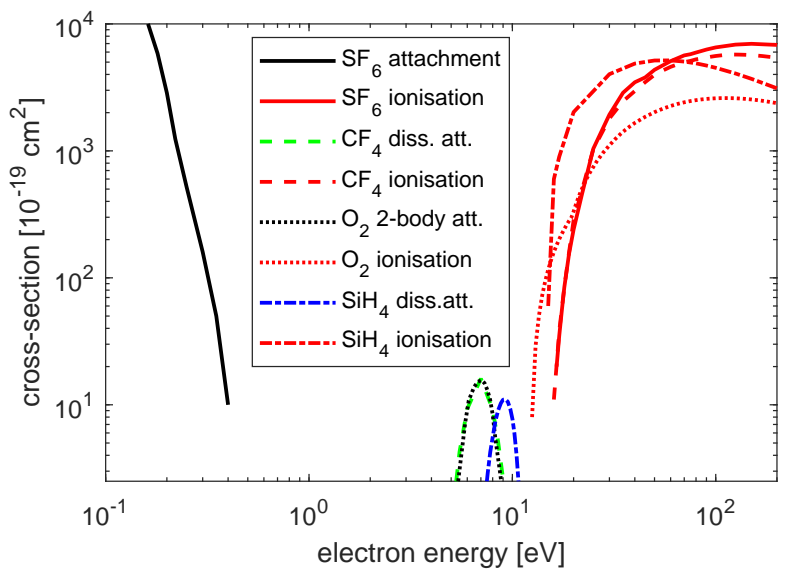

Figure 7. Ionisation and attachment cross-sections for $\mathrm{SF}_{6}, \mathrm{CF}_{4}, \mathrm{O}_{2}$, and $\mathrm{SiH}_{4}$ gases. The attachment cross-sections for $\mathrm{CF}_{4}$ and $\mathrm{O}_{2}$ are almost overlapping in the figure. 


\section{Anion reaction chemistry for small silanions $(n \leq 6)$}

Having confirmed that silanions are formed within the plasma, we can now consider how the wide range of anion clusters are formed.

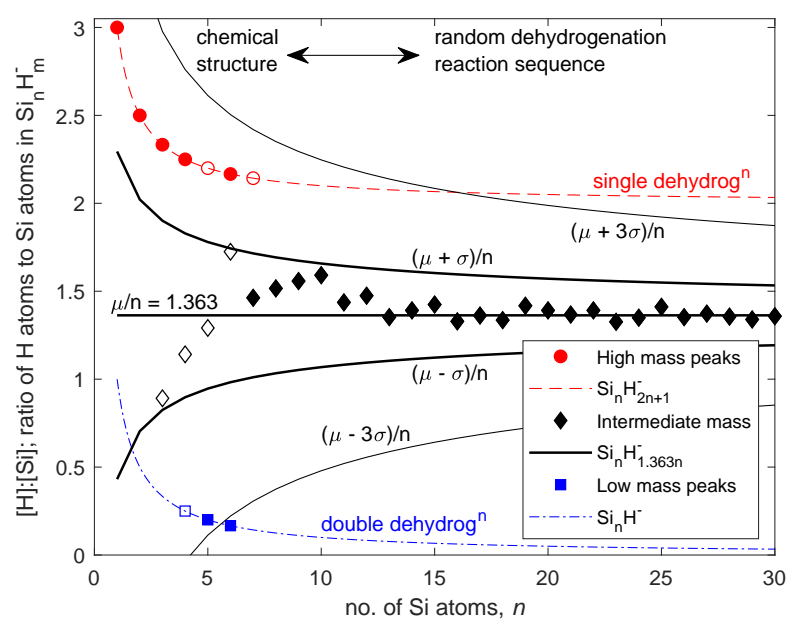

Figure 8. The ratio of hydrogen content to number of $\mathrm{Si}$ atoms, $[\mathrm{H}]:[\mathrm{Si}]$, in each $n$th silanion. Red circles: Series $\mathrm{Si}_{n} \mathrm{H}_{2 n+1}^{-}$. Red dashed line: Single dehydrogenation for odd masses. Blue squares: Series $\mathrm{Si}_{n} \mathrm{H}^{-}$. Blue dash-dot line: Double dehydrogenation for odd masses. Black diamonds: Series $\mathrm{Si}_{n} \mathrm{H}_{1.363 n}^{-}$. Full symbols indicate dominant abundance; open symbols indicate prominent abundance.

The small-molecule anions are strongly influenced by their electronic configuration. Fig. 2, resumed in Fig. 8, shows that $\mathrm{Si}_{n} \mathrm{H}_{2 n+1}^{-}$are dominant for $n=1-4$, and prominent for $n \approx 5-7$. These are iso-electronic with their corresponding neutral saturated linear molecules, the polysilanes $\mathrm{Si}_{n} \mathrm{H}_{2 n+2}$, which are analogues of the linear (or normal) alkanes $\mathrm{C}_{n} \mathrm{H}_{2 n+2}$. For example, $\mathrm{Si}_{3} \mathrm{H}_{7}^{-}$at mass 91 amu features very strongly, mimicking the saturated neutral polysilane $\mathrm{Si}_{3} \mathrm{H}_{8}$, which has almost zero electron affinity [66], as could be expected for a saturated molecule. These polysilanes are binary silicon-hydrogen compounds which have only $\mathrm{Si}-\mathrm{H}$ and $\mathrm{Si}-\mathrm{Si}$ single bonds. The simplest isomer of a polysilane is $\left(\mathrm{SiH}_{2}\right)_{n}$ in which the silicon atoms are arranged in a single chain backbone with no Si branches. However the chain of silicon atoms may also be branched at one or more points, and the number of possible isomers increases rapidly with the number of silicon atoms, analogous to the alkanes.

\subsection{Isomers, isotopologues, equal-mass silanions, and deconvolution}

What is surprising at first sight is that, despite its very small electron affinity, $\mathrm{Si}_{3} \mathrm{H}_{8}$ itself appears to form a significant flux of $\mathrm{Si}_{3} \mathrm{H}_{8}^{-}$(mass $92 \mathrm{amu}$ ) in Fig. $2^{+}$. In fact, all of the silanions $\mathrm{Si}_{n} \mathrm{H}_{m}^{-}$with mass greater than $\mathrm{Si}_{n} \mathrm{H}_{2 n+1}^{-}$i.e. heavier than $30 n+1 \mathrm{amu}$, are due to higher-mass silicon isotopes. For given $n$ and $m$ values, all $\mathrm{Si}_{n} \mathrm{H}_{m}$ anions are (structural) isomers; they have the same chemical composition although their structures

\footnotetext{
+ This error was made in Ref. [25] by postulating silanions up to $\mathrm{Si}_{n} \mathrm{H}_{2 n+3}^{-}$.
} 
can be different. They also contain a random distribution of the three stable isotopes of silicon [36]. Isomers that differ only in their isotopic composition are called isotopologues and their masses are different. The nature of quadrupole mass analysers means that all silanions with the same mass:charge ratio will contribute to the counts per second at the same number of amu (only single electron attachment is expected). Hence it is not possible to distinguish between, for example, ${ }^{28} \mathrm{Si}_{2} \mathrm{H}_{4}^{-},{ }^{28} \mathrm{Si}^{29} \mathrm{SiH}_{3}^{-},{ }^{28} \mathrm{Si}^{30} \mathrm{SiH}_{2}^{-},{ }^{29} \mathrm{Si}_{2} \mathrm{H}_{2}^{-}$, ${ }^{29} \mathrm{Si}^{30} \mathrm{SiH}^{-}$, or ${ }^{30} \mathrm{Si}_{2}^{-}$, which all have the same $60 \mathrm{amu}$ mass in the spectrum.

The isotope natural abundance can be deconvolved from the mass spectrum [36] to some limited extent (see Appendix A and Appendix B). This is shown explicitly in Fig. 9 for the smallest anions $n=1-6$, by deconvolving the isotope ratios from the measured spectrum. Small residuals due to experimental imprecision have been neglected in Fig. 9(c). Therefore, for these small silanions, the largest are indeed $\mathrm{Si}_{n} \mathrm{H}_{2 n+1}^{-}$. This concurs with Peppernick et al for $\mathrm{Si}_{n} \mathrm{H}_{m}^{-}, n=8-12$ [36]. Now that the silanions are identified unambiguously in Fig. 9(c) by their single majority isotopologue ${ }^{28} \mathrm{Si}_{n} \mathrm{H}_{m}^{-}$, their reactions can be considered.

(a) raw data, including silicon isotopes

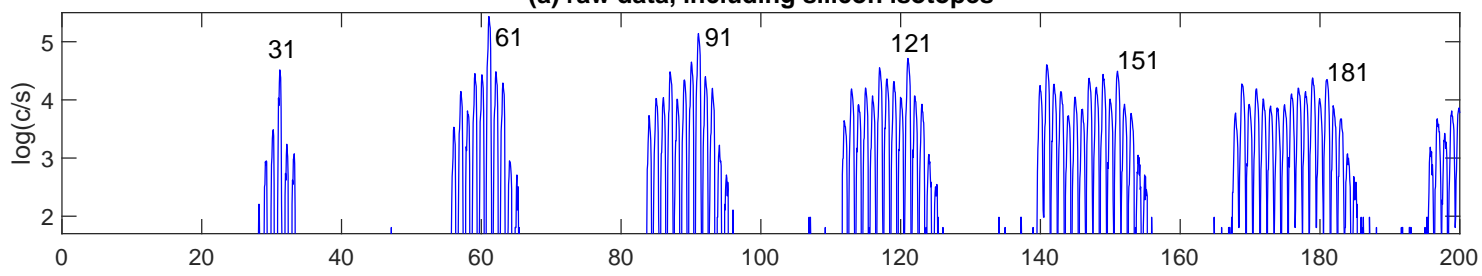

(b) discretized data, including silicon isotopes

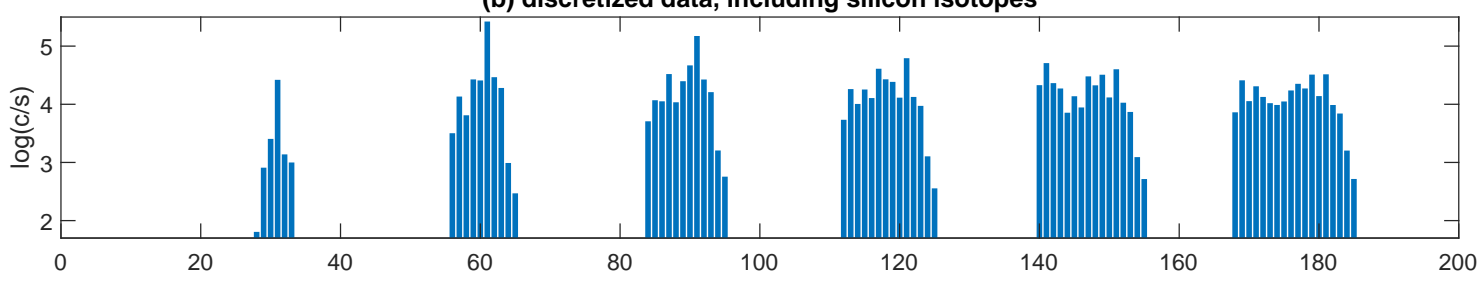

(c) deconvolved data, for isotope ${ }^{28} \mathrm{Si}$ only

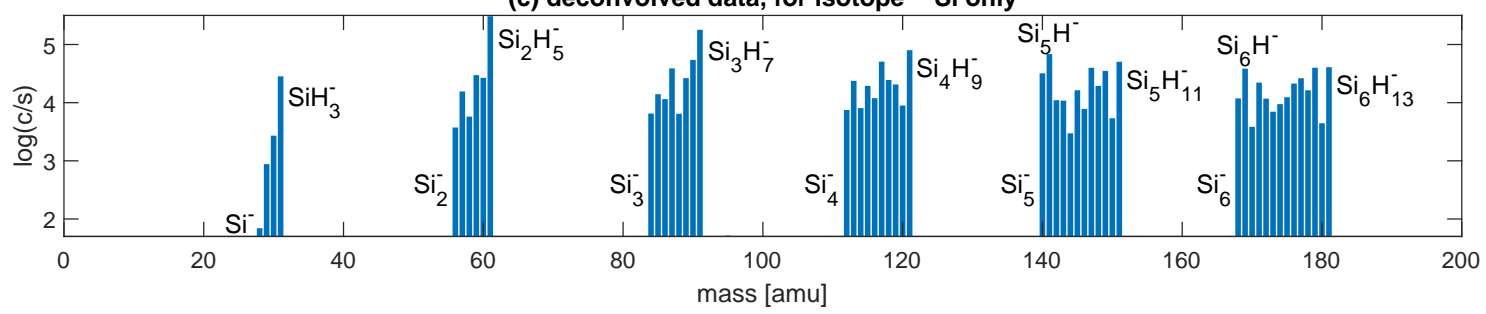

Figure 9. (a) Raw data for 0 - $200 \mathrm{amu}$, from Fig. 2. The mass numbers of some peaks are marked for reference. (b) Discretized abundance mass spectrum for the first six complete silanion clusters, by integrating the signal in (a) over the profile for each amu (renormalized to the same ordinate limits). (c) Deconvolution of the abundance mass spectrum in (b), thereby replacing the isotope abundances $\left\{{ }^{28} \mathrm{Si},{ }^{29} \mathrm{Si},{ }^{30} \mathrm{Si}\right\}$, by ${ }^{28} \mathrm{Si}$ alone. 


\subsection{Single dehydrogenation}

The simplest growth mechanism for a series of $\mathrm{Si}_{n} \mathrm{H}_{2 n+1}^{-}$silanions would be to assume sequential reactions with silane, liberating a single $\mathrm{H}_{2}[33,35]$ :

$$
\text { Single dehydrogenation series } \mathrm{Si}_{n} \mathrm{H}_{2 n+1}^{-}+\mathrm{SiH}_{4} \rightarrow \mathrm{Si}_{n+1} \mathrm{H}_{2(n+1)+1}^{-}+\mathrm{H}_{2} \cdot(1)
$$

Note that single deydrogenation, following a reaction with $\mathrm{SiH}_{4}$, has the net effect of adding one $\mathrm{Si}$ atom and two $\mathrm{H}$ atoms to the silanion. Previously only a tentative assumption [33], this reaction can now be stated with confidence, based on the observations by Operti et al [35]. For a given $n$, these are the heaviest possible silanions, as discussed above and shown in Fig. 9(c), because the heaviest possible neutral $\mathrm{Si}_{n} \mathrm{H}_{2 n+2}$ has zero electron affinity [66]. These single dehydrogenation reactions are generally included in theoretical silanion-anion nucleation models $[1,5,6,7,8,9$, $10,11,12,13,14,15,16,19,20,21,22,23,24]$.

\subsection{Double dehydrogenation}

Another prominent series in Figs. 2, 8 and 9 are the silicon mono-hydrides $\mathrm{Si}_{n} \mathrm{H}^{-}$; these anions are intense for $n=4-6$, and $\mathrm{Si}_{5} \mathrm{H}^{-}$is especially abundant [5, 35]. Measurements and $a b$ initio calculations of $\mathrm{Si}_{n} \mathrm{H}^{-}(n=2-4)$ by $\mathrm{Xu}$ et al [67] find that $\mathrm{Si}_{2} \mathrm{H}^{-}$and $\mathrm{Si}_{3} \mathrm{H}^{-}$are non-classical $\mathrm{H}$-bridged structures, whereas $\mathrm{Si}_{4} \mathrm{H}^{-}$has the traditional $\mathrm{Si}-\mathrm{H}$ single bond, as expected for $\mathrm{H}$ bonding to silicon surfaces. It is suggested that $\mathrm{Si}_{4} \mathrm{H}^{-}$ represents the transition between bridged and single $\mathrm{Si}-\mathrm{H}$ bonds [67], which could be supported by the qualitative difference between the smallest mono-hydrides and the particularly intense series $n=4-6$ in Fig. 2. Calculations by Yang et al [68] confirm and explain the $\mathrm{Si}-\mathrm{H}$ structure for $\mathrm{Si}_{n} \mathrm{H}^{-}(n=4-10)$. Apart from one single $\mathrm{H}$ atom, $\mathrm{Si}_{n} \mathrm{H}^{-}$anions are pure silicon clusters, so the double dehydrogenation series may play a role in the generation of quasi-crystalline particles [69] in diluted silane as reported by Roca i Cabarrocas et al [70, 71]. These low mass anions contribute strongly to the early cluster distributions, thereby distorting the purely-statistical distribution in Fig. 2 until $n \approx 13$. Although silicon appears directly below carbon in the periodic table, there are substantial differences in the structure of pure silicon and carbon clusters of comparable size. Silicon clusters exhibit compact, three-dimensional structures in contrast to small carbon clusters, which form linear chains and rings. This difference is attributed to the much weaker $p$ bonding in silicon clusters, thereby favoring structures with a large number of single bonds [72].

The simplest growth mechanism for a series of $\mathrm{Si}_{n} \mathrm{H}^{-}$would be to assume sequential silane reactions, liberating two $\mathrm{H}_{2}$ [33]:

$$
\text { Double dehydrogenation series } \mathrm{Si}_{n} \mathrm{H}^{-}+\mathrm{SiH}_{4} \rightarrow \mathrm{Si}_{n+1} \mathrm{H}^{-}+2 \mathrm{H}_{2} .
$$

Note that double deydrogenation, following a reaction with $\mathrm{SiH}_{4}$, has the net effect of adding one Si atom (but no $\mathrm{H}$ atoms) to the silanion. Operti et al [35] confirmed that the most frequent silanion-silane condensation steps are indeed followed by single or double dehydrogenation, the latter resulting in abundant $\mathrm{Si}_{n} \mathrm{H}^{-}(n=2-5)$ as in reaction 
(2). These results are in good agreement with the present work. In their particular conditions, even triple dehydrogenation could be observed [35]. However, a chemical kinetics network [23, 24] model of multiple dehydrogenation would rather expect (2) to involve successive steps, not a single elementary step; this is discussed further in Sec. 7. Note that ${ }^{28} \mathrm{Si}_{n} \mathrm{H}^{-}$are the lightest possible silanions having an odd mass number.

Hence the mass range for the odd-mass-number silanions goes from ${ }^{28} \mathrm{Si}_{n} \mathrm{H}^{-}$, mass $(28 n+1)$ amu, up to ${ }^{28} \mathrm{Si}_{n} \mathrm{H}_{2 n+1}^{-}$, mass $(30 n+1)$ amu. The lowest mass results from a series of purely double dehydrogenation reactions, and the highest mass results from a series of purely single dehydrogenation. Intermediate odd-mass silanions result from combinations of these two reaction series. The even-mass-number series is discussed in Sec. 6.5.

\section{Anion reaction chemistry for large silanions $(n>13)$}

The regular spectrum for large- $n$ anions in Fig. 2 suggests that cluster growth also occurs via successive silanion reactions with monomers. Operti et al [35] show that these monomers are likely to be $\mathrm{SiH}_{4}$, which, in any case, are much more numerous than the radicals $\mathrm{SiH}_{0-3}$ in these $\mathrm{RF}$ plasmas. In contrast to the specific chemical influences on small molecular silanions, the broad featureless distribution of hydrogen in large silanions, $n>13$, is indicative of purely statistical growth reactions.

\subsection{Dehydrogenation branching ratio, $f$.}

From Figs. 2 and 3, the dominant abundance within large clusters is for $\mathrm{Si}_{n} \mathrm{H}_{1.363 n}^{-}$, with a statistical distribution of $\mathrm{H}$-content around this maximum. From Fig. 8, this relative number of $\mathrm{H}$ atoms, $[\mathrm{H}]:[\mathrm{Si}]$, is clearly between the nearly-pure silicon series (double dehydrogenation) $\mathrm{Si}_{n} \mathrm{H}^{-}$, and the saturated silanion series (single dehydrogenation) $\mathrm{Si}_{n} \mathrm{H}_{2 n+1}^{-}$; i.e. $1<1.363 n<2 n+1$, for all $n \geq 1$. Following the dehydrogenation reactions demonstrated by Operti et al [35] and described in Sec. 5, it therefore seems reasonable that the large silanions grow via a random sequential mix of single and double dehydrogenation reactions.

We therefore introduce $f$ as the branching ratio for single $\mathrm{H}_{2}$ dehydrogenation, and $(1-f)$ as the branching ratio for double $\mathrm{H}_{2}$ dehydrogenation [33]. Other reactions, such as elimination of single $\mathrm{H}$ atoms, or triple dehydrogenation, are arbitrarily excluded here, on energy grounds [35]. After $n$ successive reactions with $\mathrm{SiH}_{4}$ molecules, a silanion will grow by $n$ Si-atoms; also, $4 n \mathrm{H}$-atoms will be added, minus $2 n f$ eliminated by single dehydrogenation, and $4 n(1-f)$ eliminated by double dehydrogenation. Hence, on average, the final silanion contains $4 n-2 n f-4 n(1-f)=2 n f$ H-atoms, giving $\mathrm{Si}_{n} \mathrm{H}_{2 n f}^{-}$(equivalently, because double dehydrogenation adds no $\mathrm{H}$-atoms, the only $\mathrm{H}$ contribution comes from the $n f$ reactions which each add two $\mathrm{H}$ atoms). Equating this with the experimentally-observed most probable silanion, gives $\mathrm{Si}_{n} \mathrm{H}_{2 f n}^{-} \equiv \mathrm{Si}_{n} \mathrm{H}_{1.363 n}^{-}$, hence $f=0.68$. The constant branching ratio $f$ for all clusters is a consequence of the 
measured constant gradient in Fig. 3; a possible reason for this is discussed in Sec. 7.1.

To summarize, single deydrogenation $(68 \%)$ is approximately twice as likely as double dehydrogenation (32\%) during silanion growth in these plasma conditions. Different plasma conditions would be expected to have a different branching ratio $f$, and thereby influence the composition of the clusters, as discussed in Sec. 7. The mass distributions calculated below can easily be re-calculated for any value of $0<f<1$ using Appendix B.

6.1.1. Cross-linked, three-dimensional structures. The maximum-abundance silanions $\mathrm{Si}_{n} \mathrm{H}_{1.363 n}^{-}$have $4 n \mathrm{Si}$ valence sites of which $1.363 n$ are occupied by $\mathrm{H}$. Hence there are 2.637 silicon bonds per $\mathrm{Si}$ atom, approximately $\frac{8}{3}$. Therefore, roughly, for every three $\mathrm{Si}$ atoms there are two with $3 \mathrm{Si}-\mathrm{Si}$ bonds and one with $2 \mathrm{Si}$-Si bonds (or one with 4 Si-Si bonds and two with $2 \mathrm{Si}$-Si bonds), instead of all three with just $2 \mathrm{Si}$-Si bonds as in a polysilane chain. Silanions are therefore highly cross-linked, three-dimensional structures (see Appendix C). This kind of structure was proven for pure silicon anion clusters by ab initio calculations [72].

\subsection{Binomial and Gaussian statistics for the hydrogen distribution}

A purely-descriptive random bonding theory was previously used to model mass spectra [31], but the work by Operti et al [35] means that a more meaningful statistical dehydrogenation reaction sequence can now be justified. During silanion growth, the occurrence of a single or a double dehydrogenation reaction is assumed to be a random event, with probability $f$ and $(1-f)$ respectively, independently of preceding reactions. Both odd- and even-mass silanions are present in Figs. 2 and 9(c), but only odd-mass silanions will be considered first of all, because they constitute the principal abundances amongst the initial silanions.

The probability distribution of $n$ binary choices of single or double dehydrogenations, which results in a silanion with $n \mathrm{Si}$ atoms, is described by a binomial distribution:

$$
\{f+(1-f)\}^{n}=\sum_{j=0}^{n} C_{j}^{n} f^{j}(1-f)^{(n-j)}=1,
$$

where $j$ is the number of single deydrogenations which add $j \mathrm{H}_{2}$ to $\mathrm{Si}_{n} \mathrm{H}^{-}$, resulting

in the silanion odd-mass isomers $\mathrm{Si}_{n} \mathrm{H}_{2 j+1}^{-} . \quad C_{j}^{n}=\frac{n !}{j !(n-j) !}$ is the number of isomeric combinations of $j$ single, and $(n-j)$ double, dehydrogenations. The fractional abundance of these silanions is $C_{j}^{n} f^{j}(1-f)^{(n-j)}$ which all have mass $(28 n+2 j+1)$ amu and so are indistinguishable in the mass spectrum (only the ${ }^{28} \mathrm{Si}$ isotope is considered here; higher-mass isotopes are accounted for in the following Sec. 6.3). Note that the sum of the binomial probabilities is unity for each cluster because $\{f+(1-f)\}^{n}=1$ in (3).

The probability of $n$ consecutive single dehydrogenations (1), yielding the heaviest silanions $\mathrm{Si}_{n} \mathrm{H}_{2 n+1}^{-}$, is $f^{n}$, starting with the mono-silanion $\mathrm{SiH}_{3}^{-}(n=1 ; j=1)$. 
The probability of $n$ consecutive double dehydrogenations (2), yielding the lightest odd-mass silanions $\mathrm{Si}_{n} \mathrm{H}^{-}$, is $(1-f)^{n}$, starting with the mono-silanion $\mathrm{SiH}^{-}(n=1 ; j=$ $0)$.

For $n \gg 1$, the most probable number of $\mathrm{H}_{2}$ (or more correctly, the number of $2 \mathrm{H})$ in the silanion is the same as the binomial average number, the mean $\mu_{\mathrm{H}_{2}}=n f$. In terms of amu, therefore, the mean number of $\mathrm{H}$ atoms is $\mu=2 \mu_{\mathrm{H}_{2}}=2 n f$ amu. Equating with the most abundant silanions observed gives $2 n f=1.363 n$ as before. The binomial distribution is shown in Fig. 10 for the odd-H distribution in the pure isotope ${ }^{28} \mathrm{Si}_{19} \mathrm{H}_{2 j+1}^{-}$using $f=0.68$. The distribution is skewed to higher $\mathrm{H}$ content because $f>0.5$, and the fractional abundance of the mono-hydride silanion is vanishingly small, $(1-f)^{19}=0.32^{19}=4 \cdot 10^{-10}$.

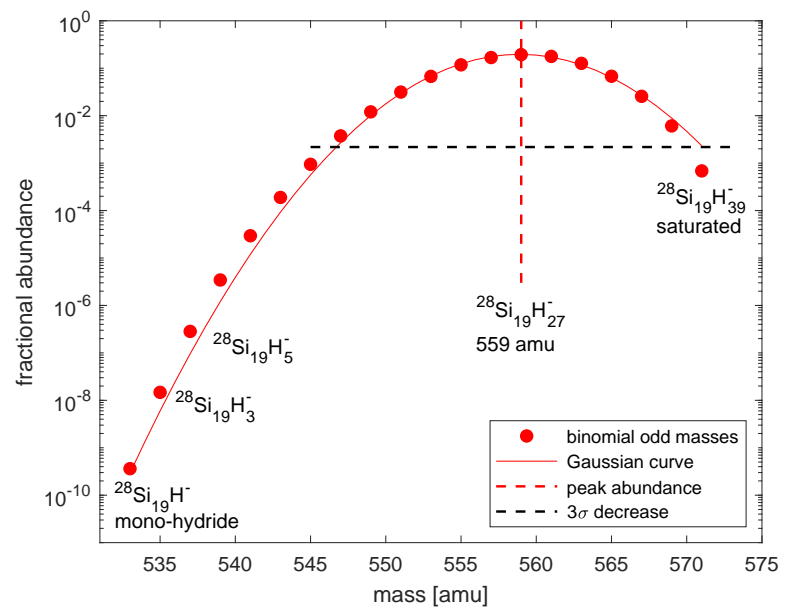

Figure 10. Binomial distribution, for $f=0.68$, of the $\mathrm{H}$ content in ${ }^{28} \mathrm{Si}_{19} \mathrm{H}_{2 j+1}^{-}$ $(j=0-19)$ for the odd-mass silanions. The maximum abundance at ${ }^{28} \mathrm{Si}_{19} \mathrm{H}_{27}^{-}$is the closest odd-mass to the peak ${ }^{28} \mathrm{Si}_{19} \mathrm{H}_{(1.363 \times 19)}^{-}$corresponding to the fit in Fig. 3. The full range is shown, from the smallest silanion (mono-hydride ${ }^{28} \mathrm{Si}_{19} \mathrm{H}^{-}$) to the largest silanion, ${ }^{28} \mathrm{Si}_{19} \mathrm{H}_{(2 \times 19+1)}^{-}$, in the odd-mass series for $n=19$.

Concerning the width of the clusters' mass distribution, the variance of the binomial distribution for the number of $\mathrm{H}_{2}$ is $\sigma_{\mathrm{H}_{2}}^{2}=n f(1-f)$, where $\sigma_{\mathrm{H}_{2}}$ is the standard deviation from the mean. In terms of amu, the standard deviation is therefore the double, $\sigma=2 \sigma_{\mathrm{H}_{2}}=2 \sqrt{n f(1-f)}$ amu. For Fig. $10, \sigma \approx 4 \mathrm{amu}$, and so the mass difference between the $3 \sigma$ points is $\approx 24$ amu. The standard deviation relative to the cluster number $n$ in Fig. 8 is $\frac{\sigma}{n}=2 \sqrt{\frac{f(1-f)}{n}}$, which becomes narrower as the cluster size increases.

6.2.1. Gaussian distribution Generally, a binomial distribution can be approximated by a Gaussian distribution for $n \gg 1$ and $f$ not too different from $\frac{1}{2}$. This is shown in Fig. 10 by superposing a normalized Gaussian, namely $\frac{1}{\sigma_{\mathrm{H}_{2}} \sqrt{2 \pi}} \exp \left[-\frac{1}{2}\left(\frac{x-\mu_{\mathrm{H}_{2}}}{\sigma_{\mathrm{H}_{2}}}\right)^{2}\right]$, where $x$ is the number of $\mathrm{H}_{2}$, and the mean $\mu_{\mathrm{H}_{2}}$ and standard deviation $\sigma_{\mathrm{H}_{2}}$ are identical to the binomial parameters used above. Gaussian curves are (symmetric) parabolas in the 
semilog plot, so the fit to the skewed binomials is less good near the extreme values. The abundance fraction at three standard deviations from the mass peak is $\mathrm{e}^{-\frac{9}{2}}=0.011$ of the maximum (the black dotted line in Fig. 10). For higher abundances, the distribution appears to be almost symmetrical around the peak mass. Again, the integrated area below the whole Gaussian curve is unity, and the maximum probability $\frac{1}{\sigma_{\mathrm{H}_{2}} \sqrt{2 \pi}}(\approx 0.2$ in Fig. 10) decreases proportionally to $\frac{1}{\sqrt{n}}$.

\subsection{Silicon isotope distribution and iterative convolution}

The isotope abundance can be directly deconvolved from the mass spectrum [36] (see Appendix A and Appendix B), but above $n \approx 6$, it is necessary, instead, to convolve the binomial distribution with the isotope abundance so that the model distribution can be compared with the measured mass spectrum in Fig. 2. Note that, if the Si isotope is not stated explicitly, then $\mathrm{Si}_{n} \mathrm{H}_{m}^{-}$refers to the average silicon isotopic mass $28.109 \mathrm{amu}$, as in Sec. 2.

It is reasonable to expect that the probabilities of dehydrogenation reactions are entirely unaffected by the different silicon isotopes, and therefore the isotopic distribution can be calculated independently of the binomial distribution for the $\mathrm{H}$ content in Sec. 6.2.

By analogy with the binomial expansion for the binary choice of single or double dehydrogenation, the triple isotope distribution can be described by a trinomial expansion, as described in Appendix D. However, it is necessary to collect terms having the same mass. In this case, it is more convenient to compute iterated convolutions of the three isotopes (see Appendix B) than to expand the trinomial.

Iterated convolution is equivalent to repeated polynomial multiplication. To calculate the abundances of the monomer $\mathrm{Si}$, the isotope fractional abundance array iso $=[0.9221,0.0470,0.0309]$ is convolved with unity to obtain the same array for the masses $\{28,29,30\}$ amu, by definition, representing $\left\{{ }^{28} \mathrm{Si},{ }^{29} \mathrm{Si},{ }^{30} \mathrm{Si}\right\}$, respectively. The abundances of $\mathrm{Si}_{2}$ are obtained by a second convolution with iso to obtain iso $*$ iso= $i s o^{* 2}=[0.8503,0.0867,0.0592,0.0029,0.0010]$ for the masses $\{56,57,58,59,60\} \mathrm{amu}$, representing $\left\{{ }^{28} \mathrm{Si}_{2},{ }^{28} \mathrm{Si}^{29} \mathrm{Si}^{29}{ }^{29} \mathrm{Si}_{2} \&{ }^{28} \mathrm{Si}^{30} \mathrm{Si}^{29}{ }^{29} \mathrm{Si}^{30} \mathrm{Si}^{30}{ }^{30} \mathrm{Si}_{2}\right\}$, respectively. $n$ successive convolutions with iso (mathematical notation $i s o^{* n}$ ), yields the abundances of the $2 n+1$ masses of $\mathrm{Si}_{n}$, beginning with mass $28 n$ and ending with $30 n$ amu. The sum of the abundances for each $\mathrm{Si}_{n}$ is always unity, and the average mass is always $28.109 n$ amu. These silicon isotopologue distributions are shown in Fig. 11 and are verified to be identical to the trinomial expansion results. Note that these silicon isotope distributions are not intended as pure $\mathrm{Si}_{n}$ clusters; instead, they represent the isotopic mass distribution of the $n \mathrm{Si}$ atoms in the isotopologues of $\mathrm{Si}_{n} \mathrm{H}_{m}^{-}$isomers. Although the

${ }^{28} \mathrm{Si}$ isotope is strongly dominant in the low- $n$ series, the abundance of the pure isotope mass $28 n$ becomes outweighed by the total probabilities of multiple isotope combinations [36] around $n>19$, which shifts the isotopologue mass peak to higher and higher values. 

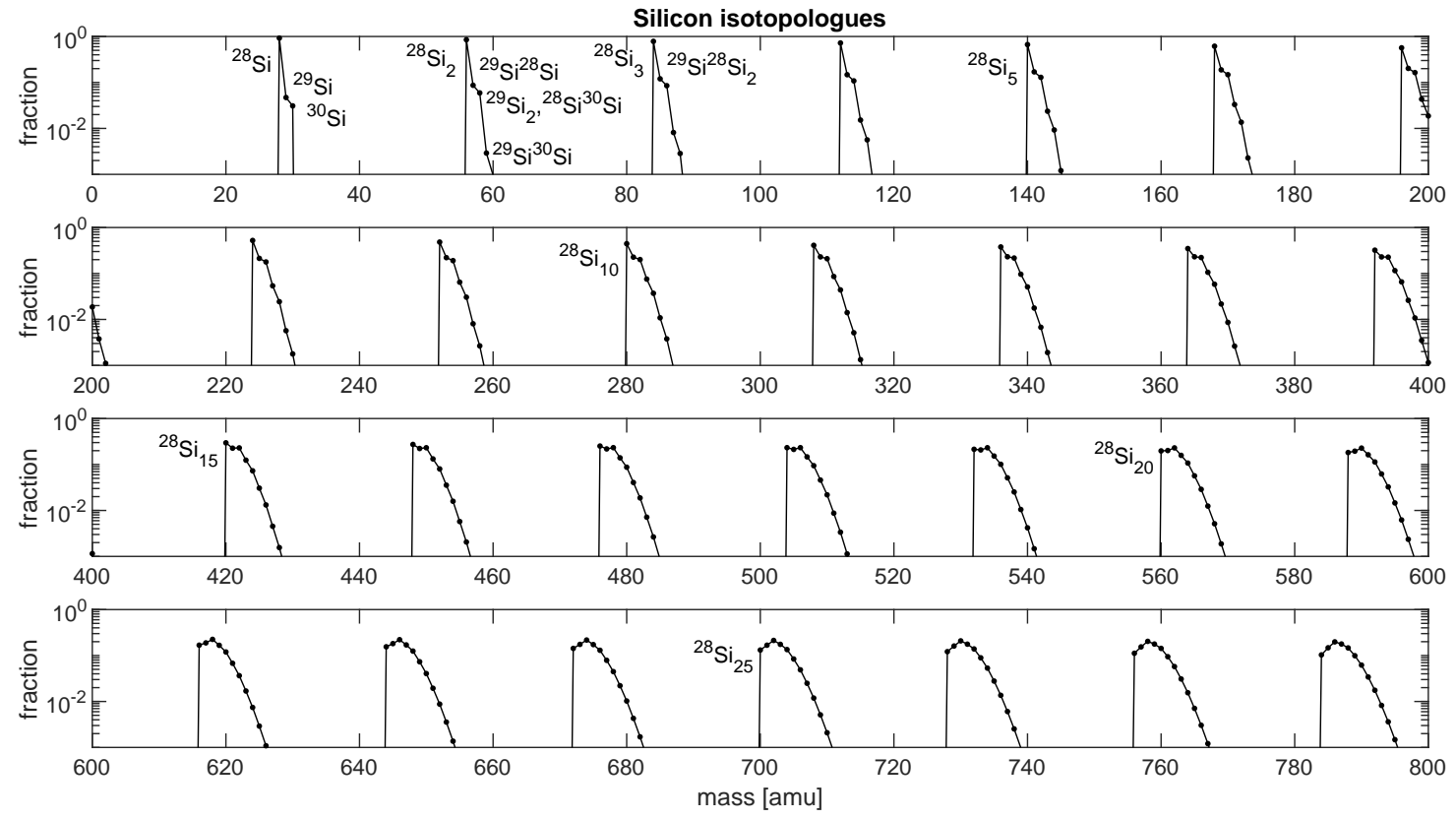

Figure 11. The fractional abundances of the combinations of the three Si isotopes, for each $\mathrm{Si}_{n}$ which occur in $\mathrm{Si}_{n} \mathrm{H}_{m}^{-}$. The mass ranges, and dynamic range of the $y$-axis, are similar to Fig. 2, and some isotopologues have been labelled where space permits, before the number of isotope permutations becomes too large to label. The masses range from $28 n$ amu for pure ${ }^{28} \mathrm{Si}_{n}$ (fraction $0.9221^{n}$ ), up to $30 n$ amu for pure ${ }^{30} \mathrm{Si}_{n}$ (fraction $\left.0.0309^{n}\right)$. There are therefore $(2 n+1)$ mass values for each $\mathrm{Si}_{n}$, although the fractions of the higher-isotope combinations are too small to appear in this figure. The sum of all the fractions equals one for each cluster.

\subsection{Convolution of the H binomial distribution with the Si isotope distribution}

Because the distributions of $\mathrm{H}$ content and $\mathrm{Si}$ isotopes are independent of each other, their combination is given by a direct convolution (Appendix B) of the binomial distributions in Sec. 6.2, with the isotopologue distributions in Sec. 6.3. The binomial (red points) and Gaussian (red line) distributions in Fig. 12(a) show the odd mass series in the range $400-600 \mathrm{amu}$, covering the $n^{\text {th }}$ silanions from ${ }^{28} \mathrm{Si}_{14} \mathrm{H}_{2 j+1}^{-}$to ${ }^{28} \mathrm{Si}_{20} \mathrm{H}_{2 j+1}^{-}$ for $j=0-n$. Fig. 12(a) therefore includes the ${ }^{28} \mathrm{Si}_{19} \mathrm{H}_{2 j+1}^{-}$distribution of Fig. 10, but only shows those masses within $3 \sigma$ of the peak value which have more than $\mathrm{e}^{-\frac{9}{2}}=1.1 \%$ of the maximum abundance (i.e. above the black horizontal line in Fig. 10). The sum over all the fractions of each binomial odd-mass silanion is unity.

Convolution with the isotopic distribution results in the points joined by the black line in Fig. 12(a). The most salient feature is the sawtooth appearance on its rising flank, owing to the isotopologues in Fig. 11 being skewed uniquely to higher masses, ${ }^{29} \mathrm{Si}$ and ${ }^{30} \mathrm{Si}$. This asymmetry of the isotope abundance gives some even-mass signal by 'isotopic scrambling': Specifically, convolution with ${ }^{29} \mathrm{Si}$ means that the oddmass silanions do contribute to even mass numbers. The sum of abundances for the binomial distributions, and for the convolved distributions, are individually unity for each silanion; the convolved abundances are lower because they are distributed over 

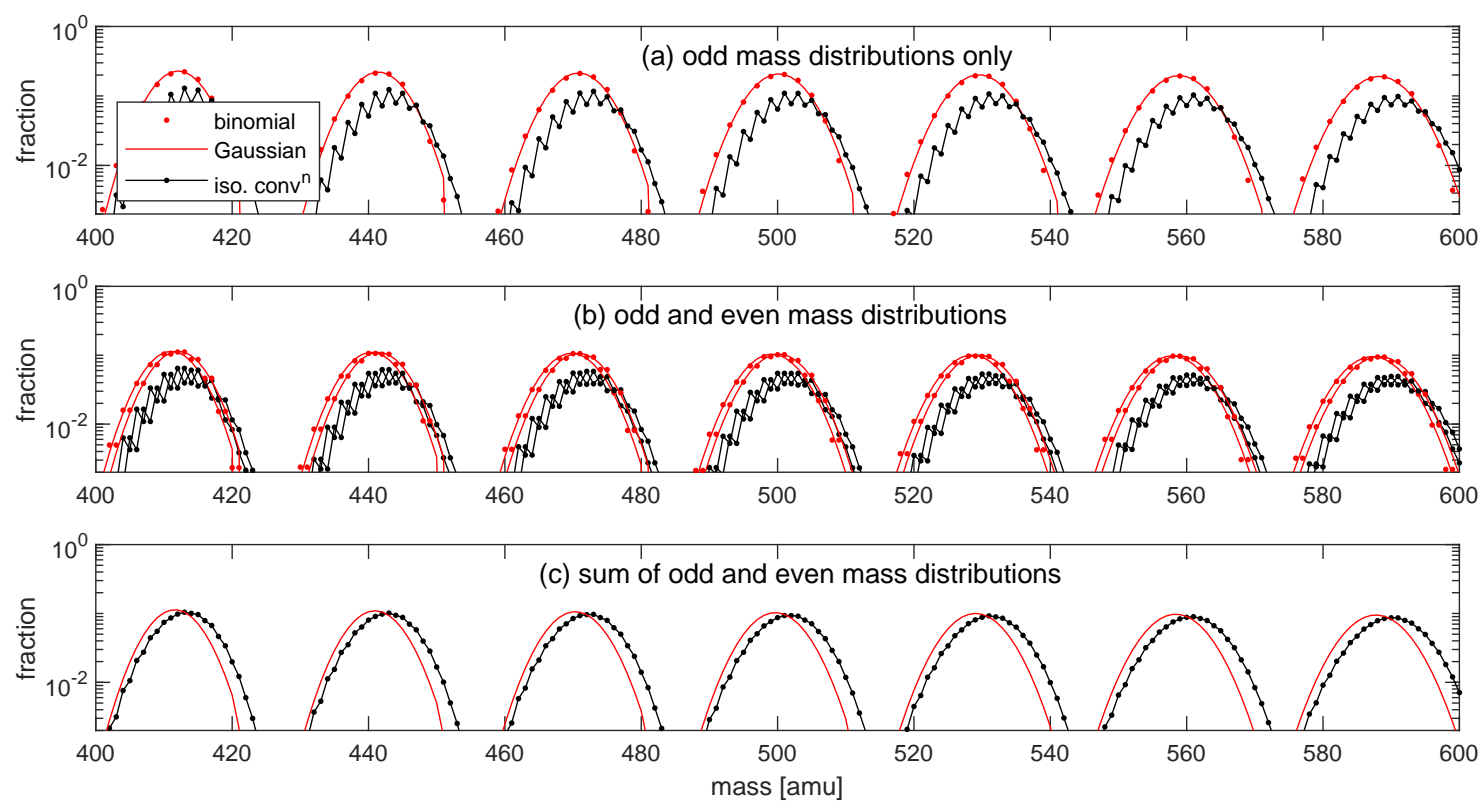

Figure 12. Calculated binomial (red points) and Gaussian (red line) distributions, and convolution with the silicon isotopes (black points and line), in the range 400-600 amu for (a) odd-mass silanions only; (b) for individual odd- and even-mass silanions; and (c) the sum of the convolved distributions for odd and even masses. Convolution with the silicon isotope distribution skews the binomial and Gaussian curves to higher masses.

every mass, and not just the odd masses.

\subsection{Odd and even mass series}

The measured mass spectrum in Fig. 2 does not exhibit the odd-even sawtooth appearance in Fig. 12(a). Clearly, there must also be an even-mass series, $\mathrm{Si}_{n}^{-}$to $\mathrm{Si}_{n} \mathrm{H}_{2 n}^{-}$, because these already exist in the initial anions in Fig. 9(c). The corresponding even-mass dehydrogenation reactions,

$$
\text { Single dehydrogenation even series } \mathrm{Si}_{n} \mathrm{H}_{2 n}^{-}+\mathrm{SiH}_{4} \rightarrow \mathrm{Si}_{n+1} \mathrm{H}_{2(n+1)}^{-}+\mathrm{H}_{2},(4)
$$

and

$$
\text { Double dehydrogenation even series } \mathrm{Si}_{n}^{-}+\mathrm{SiH}_{4} \rightarrow \mathrm{Si}_{n+1}^{-}+2 \mathrm{H}_{2} \text {, }
$$

exist in parallel with the odd-mass dehydrogenation (1) and (2). The odd-mass reactions (1) and (2) dominate for the small anions in Sec. 5, but this discriminating influence of the chemical structure disappears for the large silanions. All other particularities of the small anions, such as the remarkable emergence of $\mathrm{Si}_{5} \mathrm{H}^{-}$and $\mathrm{Si}_{6} \mathrm{H}^{-}$, are also eventually lost in the statistical averaging of iterated growth reactions.

Furthermore, the even-mass series must have abundance and branching ratio $f$ closely equal to the odd-mass series, otherwise there would always be some residual odd-even alternation in the model, which is not observed amongst the large silanions in Fig. 2. Some coupling across the odd- and even-mass series could occur via occasional 
elimination [35] or abstraction of $\mathrm{H}$ atoms (as opposed to purely molecular $\mathrm{H}_{2}$ in dehydrogenation reactions), and/or possible reactions with minority monomer radicals $\mathrm{SiH}_{0-3}$, instead of $\mathrm{SiH}_{4}$. The constancy of the branching ratio across all odd and even masses is tentatively explained by localized reactions in Sec. 7.1.

Fig. 12(b) shows a 50/50 superposition of equal odd- and even-mass curves corresponding to those in Fig. 12(a). Finally, the points joined by the black line in Fig. 12(c) represent the sum of the convolved odd- and even-mass series. In conclusion, the skewed isotopic distributions in Fig. 11 shift the binomial curves to significantly higher masses in Fig. 12(c), showing that the isotope effect cannot be ignored if the model is to be compared with experimental results. The convolution of the Gaussian curves with the isotopologues also broadens the distributions but these are no longer Gaussian because the isotope distributions are asymmetric, giving a non-symmetric resultant curve, although it superficially resembles a Gaussian curve [36] in Fig. 12(c). Nevertheless, the area below the convolved curve remains unity because each of the Gaussian and isotope curves is normalized. The mass of the convolved maxima follows the isotopic average 29.472n amu as determined in Fig. 3.

\section{Comparison with the measured mass spectrum}

This final convolved distribution in Fig. 12(c) is compared with the entire measured mass spectrum in Fig. 2. The logarithmic slope of the measured cluster abundances is much steeper than for the purely-statistical $\frac{1}{\sqrt{n}}$ gradual fall-off in Fig. 12 because the mass-dependent silanion flux signal also depends on the combined effect of their plasma generation (some function of $t_{\text {on }}$ and $t_{\text {off }}$, see Sec. 2.3), the mass discrimination through the PPM421, and the detection sensitivity by the channeltron [33]. This is accounted for by multiplying the model curve by an empirical factor $10^{-0.0029 M}$, where $M$ is the mass in amu. These three effects are unknown functions, therefore the observed logarithmic decrement cannot be interpreted in any meaningful way.

Finally, for high mass clusters particularly, the calculated curves give an accurate fit to the measured abundance distributions to about 3 standard deviations either side of the cluster maxima, thus supporting the assumptions of the random dehydrogenation model. The mass distributions of the initial small clusters are not captured by the model because the particular chemical effects considered in Sec. 5 are not accounted for. The single and double deydrogenation model also does not include the triple hydrogenation necessary to explain the remarkable mono-hydrides $\mathrm{Si}_{5} \mathrm{H}^{-}$and $\mathrm{Si}_{6} \mathrm{H}^{-}$ [35]. Nevertheless, apart from the empirical log slope, the branching ratio between single and double dehydrogenation, $f=0.68$, is the only parameter required to fit the mass values, the distribution shape, and the width of every large silanion distribution $(n>13)$.

The determining value of $f$ in other silane experiments will differ depending

on the plasma parameters used. Almost-pure crystalline silicon clusters [69] are obtained for other experimental conditions [36], for example by silane dilution [70, 71]. 
Comparatively-low pressure experiments [35] also apparently favour a low $\mathrm{H}$ content commensurate with $f \ll 1$. An example of these cases, in the limit $f=0$ (double deydrogenation only), is shown for $\mathrm{Si}_{19} \mathrm{H}_{m}^{-}$in Fig. 13. The value $f=0.68$ in the present experiments is higher probably because of the low-duty-cycle power modulation; $f$ is likely to be a function of $t_{\text {on }}$ and $t_{\text {off }}$, which remains to be tested.

In contrast, the distribution consistent with uniquely-single dehydrogenation, $f=1$ in Fig. 13, as generally assumed in theoretical models $[1,5,6,7,8,9,10,11,12,13$, $14,15,16,17,18,19,20,21,22,23,24]$, is clearly very different from those observed by experiments. Amongst these models, double dehydrogenation is discussed apparently only by Perrin et al via two-step collisions [5] and/or reactions with silane radicals $[7,10]$. Multiple dehydrogenation is considered to be less important on energy grounds [14]. Indeed, quantum chemistry calculations propose that even single dehydrogenation involves a three-step mechanism with large energy barriers [23, 24]. Hence singlestep double dehydrogenation (which, by the microscopic reversibility principle, would furthermore be as rare as three-molecule collisions) is not expected to occur, contrary to (2). Nevertheless, the empirical fact remains that multiple dehydrogenation, via however many elementary steps, is apparently commonly observed in a wide range of plasma conditions, from low to high pressure $[5,25,26,27,29,33,35,36,42,43]$. Fridman et al [8] suggest that vibrationally-excited states in plasma could stimulate silanion-silane reactions. Also, if multiple dehydrogenation turns out to be the outcome of the reaction of an excited silanion in Operti et al [35], possibly due to ineffective quenching by the helium buffer gas in some cases, this excitation is likely to be the same as in a RF plasma, thereby explaining the agreement with the present data [41]. This emphasizes the necessity of accounting for the plasma environment in silanion-silane nucleation models.

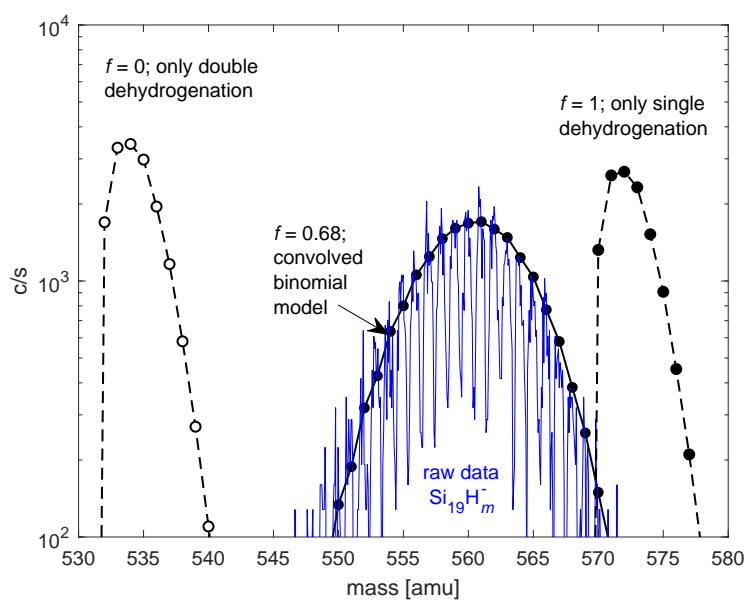

Figure 13. Raw data (blue line) are shown for $\mathrm{Si}_{19} \mathrm{H}_{m}^{-}$(taken from Fig. 2; data from neighbouring silanions are suppressed for clarity) along with the binomial model, $f=0.68$, convolved with the isotope distribution. The distribution for $f=0$ would be representative of an experiment generating crystalline clusters via double dehydrogenation. The distribution for $f=1$ represents single dehydrogenation only, as assumed in models of nucleation. 


\subsection{Constancy, statistics, and localized reactions}

Experimental measurements and comparison with the model show the following related observations:

(i) A constant ratio [H]:[Si] for all of the most-abundant silanions (Figs. 3 and 8).

(ii) A constant branching ratio $f$ of the single/double dehydrogenation reactions.

(iii) Equality of the odd- and even-mass reactions.

(iv) The accuracy of the binomial fit for the mass, shape and width of the $\mathrm{H}$ distribution (after convolution for the isotopes).

These observations could be explained by postulating localized reactions of $\mathrm{SiH}_{4}$ with only a limited number of neighbouring surface atoms on the silanions. Hence, reactions do not depend on the growth history, the size, the overall $[\mathrm{H}]:[\mathrm{Si}]$ ratio, or the odd/even number of $\mathrm{Si}$ atoms on the rest of the silanion. Single or double dehydrogenation reactions are independent of whether the preceding reaction was single or double dehydrogenation. The number of neighbouring atoms involved in the localized reactions can perhaps be judged from the size of anions where chemical influence is strong: This would suggest $n<6$ atoms of Si and H, from Figs. 2 and 9.

Silanions are charged by a single attached electron due to their non-zero electron affinity; this is qualitatively different from the dynamic equilibrium of fluctuating charges on macroscopic particles due to the plasma [29]. There is also the intriguing possibility of enhanced electron attachment to excited states of silane [73, 74]. Operti et al [35] propose that an intermediate anion-dipole complex $[47,75]$ forms by anion addition to the induced positive pole of $\mathrm{SiH}_{4}[1,10]$, i.e. to the silicon atom. Silanion-silane selfcondensation then ensues, with multiple dehydrogenation. We could further speculate that silanion-molecule reactions are a microcosm analogue for silicon thin film growth, whose surface is negatively charged in presence of a plasma sheath, and whose hydrogen content is only a few atomic per cent, which is to say, strongly dehydrogenated.

\section{Conclusions}

Mass spectra of silicon hydride negative ion clusters (defined here as "silanions", $\mathrm{Si}_{n} \mathrm{H}_{m}^{-}$) were measured up to $800 \mathrm{amu}$ in a low pressure $(0.1 \mathrm{mbar})$ pure silane plasma. Timeresolved anion flux measurements, compared with other electronegative gases, show that silanions originate from within the plasma, and not from the afterglow nor surface reactions.

The hydrogen distribution in small silanions, $n=1$ to 6 , was investigated by silicon isotope deconvolution of the raw data. These were compared with ab initio calculations and other experimental observations of silanion-silane self-condensation reactions where one, two, or three $\mathrm{H}_{2}$ molecules are released. This is an example of multiple dehydrogenation [35].

Large anions, $n>13$, were analyzed by isotopic convolution of a binomial

dehydrogenation model, for comparison with the raw data. The position, width, and 
shape of all the measured distributions were reproduced using only one fitted parameter, the branching ratio $f=0.68$ between random single and double dehydrogenation. This supports the statistical dehydrogenation model which is based on localized anion-neutral reactions. Other plasma conditions would be expected to exhibit different branching ratios.

To conclude, it is proposed that the growth mechanism of large silanions is a random sequence of multiple dehydrogenation reactions, for parallel series of odd- and evenmass silanions. Silanion-neutral nucleation can play a significant role in micro-particle formation during thin-film silicon plasma deposition. Although the theory for silanionsilane dehydrogenation reactions in plasma is still incomplete, it is suggested that nucleation models would represent experimental observations more fully by accounting for the plasma environment and including multiple dehydrogenation reactions.

\section{Acknowledgments}

We thank Professor R. Rabezzana, Università di Torino, Italy, for helpful and instructive comments which have been incorporated into the manuscript. This work was funded by Swiss Federal Research Grant Nos. BBW.EG(91)3 and BBW 93.0136 (for BRITE/EURAM Contract Nos. BE-4529-90 and BE-7328 respectively), EFREN(91)031, and BEW 9400051.

\section{Appendix A. Deconvolution of the isotope distribution}

Ideally, the whole measured mass spectrum would be deconvolved by the Si isotope distribution to facilitate interpretation in terms of anion chemistry. The confusion caused by isotope scrambling is because the signal from a single heavy isotope ${ }^{29} \mathrm{Si}$ or ${ }^{30} \mathrm{Si}$ could be mistaken for an extra $\mathrm{H}$ or $\mathrm{H}_{2}$, respectively, and so on for multiple isotope combinations. Deconvolution can be done for perfect isotope proportions, such as the mathematical model used to generate Fig. 11. However, due to experimental errors, this can be partially successful only for the smallest anions. For example, Fig. 9 shows that mass peaks above $\mathrm{Si}_{n} \mathrm{H}_{2 n+1}^{-}$are artefacts due to heavy isotopes. Unfortunately, deconvolution is impractical for larger silanions because $n$ iterated deconvolutions are required, compounding the experimental measurement errors, and the signal noise for the lowest abundances means that the residue errors are large and non-physical.

One way to perform deconvolution is to iteratively subtract the heavy isotopes starting from the unambiguous lowest-mass pure silicon isotope cluster ${ }^{28} \mathrm{Si}_{n}^{-}[36,67]$. For example, starting with the mono-silanions, the peak at mass 28 amu can only be due to ${ }^{28} \mathrm{Si}^{-}$. If silicon consisted only of this pure isotope, then the signal at $28 \mathrm{amu}$ would be augmented by dividing by its fractional natural abundance 0.9221, and the signals at 29 and $30 \mathrm{amu}$ would be diminished by subtracting the abundance fractions 0.0470 and 0.0309 of this value, respectively. This procedure is then repeated using the corrected signal at $29 \mathrm{amu}$, and so on, up to the heaviest pure-isotope mono-silanion 
${ }^{28} \mathrm{SiH}_{3}^{-}$as shown in Fig. 9.

The calculation is more complicated for the di-silanions because of the different combinations of the isotopes [36]. In fact, the masses of the $\mathrm{Si}_{2}^{-}$isotopes range from 56 amu for ${ }^{28} \mathrm{Si}_{2}^{-}$, up to 60 amu for ${ }^{30} \mathrm{Si}_{n}^{-}$, with abundances corresponding to the five terms in the convolution of the isotope probability distribution with itself, namely $[0.9221,0.470,0.0309]^{* 2}=[0.8503,0.0867,0.0592,0.0029,0.0010]$.

To generalize: For a silanion containing $n$ Si atoms, the abundance fraction of

${ }^{28} \mathrm{Si}_{n}$ is $0.9221^{n}$, and the abundances of the $(2 n+1)$ silicon isotope combinations, from $28 n$ amu up to $30 n$ amu, are given by the $n$th convolution of the abundance array $[0.9221,0.470,0.0309]^{* n}$. The iterative deconvolution calculation and results are shown respectively in Appendix B and Fig. 9 for $n=1-6$.

\section{Appendix B. Program Listings using MatlabC $[76]$}

Appendix B.1. Calculating deconvolution in Sec. 5.1

iso $=[0.9221,0.047,0.0309] ; \%$ Array of Si isotope abundances for 28,29,30 amu.

$\% \mathrm{~A}$ is the array of discretized abundance data in Fig 9(b).

$\mathrm{C}=1 ; \%$ Begin the convolution iteration with unity.

for $n=1: 6 \%$ Count through the first 6 silanions.

$\mathrm{c}=\operatorname{conv}(\mathrm{c}, \mathrm{iso}) ; \%$ Iterative convolutions of iso, giving $i s o^{* n}$.

$\%$ Note: length $(\mathbf{c})=2 n+1$, and $\operatorname{sum}(\mathbf{c})=1$ for all $n$.

for $\mathrm{j}=28^{*} \mathrm{n}: 30^{*} \mathrm{n}+1 \%$ Count through the $\mathrm{H}$ distribution in $\mathrm{Si}_{n}$ to $\mathrm{Si}_{n} \mathrm{H}_{2 n+1}$.

$\mathrm{A}(\mathrm{j})=\mathrm{A}(\mathrm{j}) / \mathrm{c}(1) ; \%$ Correction to the ${ }^{28} \mathrm{Si}$ abundance.

for $\mathrm{i}=1: 2^{*} \mathrm{n} \%$ Count through the remaining $2 n$ terms of $c$.

$A(j+i)=A(j+i)-c(i+1)^{*} A(j) ; \%$ Subtract minor isotopes from the next $2 n$ masses.

end, end, end

$\% \mathbf{A}$ is now the array of deconvolved abundance used in Fig $\mathbf{9}(\mathbf{c})$.

Appendix B.2. Calculating convolved binomial distributions in Sec. 6

iso $=[0.9221,0.047,0.0309] ; \%$ Array of Si isotope abundances for 28,29,30 amu.

nmax $=27 ; \%$ Arbitrary highest no. of Si atoms in a silanion.

Mass $=\left[1: 32^{*} \mathrm{nmax}+1\right] ; \%$ Mass array [amu]. The heaviest silanion is ${ }^{30} \mathrm{Si}_{n \max } \mathrm{H}_{2 n \max +1}^{-}$. Aiso=zeros $\left(1,32^{\star} n m a x+1\right) ; \%$ Initialize the isotopologue abundance array. $\%$ Initialize also the even- and odd-mass binomial abundances Aebin, Aobin, and the final isotope-convolved binomial abundances $A e$ and Ao, respectively.

$f=1.363 / 2 ; \%$ Single dehydrogenation branching factor, to fit the measured mass spectrum in Fig. 2, using Fig. 3.

$\mathrm{e}=0.5 ; \%$ Proportion of even masses, assumed equal to the odd masses here.

$\mathrm{c}=1 ; \%$ Begin the convolution iteration with unity.

for $\mathrm{n}=1$ :nmax \% Count through the silanion clusters from 1 up to $\mathrm{Si}_{n \max }$.

$\mathrm{c}=\operatorname{conv}(\mathrm{c}, \mathrm{iso}) ; \%$ Iterative convolutions of iso, giving $i s o^{* n}$. 
$\%$ Note: length $(\mathrm{c})=2 n+1$, and $\operatorname{sum}(\mathrm{c})=1$ for all $n$.

Aiso $\left(28^{\star} \mathrm{n}: 30^{\star} \mathrm{n}\right)=$ Aiso $\left(28^{\star} \mathrm{n}: 30^{\star} \mathrm{n}\right)+\mathrm{c}$; \% Isotopologues $\mathrm{Si}_{n}$, as used in Fig. 11.

for $\mathrm{i}=1: 2: 2^{*} \mathrm{n}+1 \%$ Index counter for hydrogenation by one $\mathrm{H}_{2}$ at a time. $\mathrm{j}=(\mathrm{i}-1) / 2 ; \%$ Number of $\mathrm{H}_{2}$ on the $n$th silanion.

$\operatorname{bin}(i)=$ factorial $(n)^{\star} f^{\wedge} j^{*}(1-f)^{\wedge}(n-j) /$ factorial $(n-j) / f a c t o r i a l(j) ; \%$ Binomial abundance, using (3).

end

Aebin $\left(28^{*} n: 30^{*} n\right)=$ Aebin $\left(28^{*} n: 30^{*} n\right)+e^{*}$ bin; \% Even mass binary abundance used in Fig. 12 .

Aobin $\left(28^{*} n+1: 30^{*} n+1\right)=$ Aobin $\left(28^{*} n+1: 30^{*} n+1\right)+(1-e)^{*}$ bin; \% Odd mass binary abundance used in Figs. 10 and $\mathbf{1 2 .}$

biniso $=\operatorname{conv}(\mathbf{c}$, bin); $\%$ Convolution of the binomial distribution with the isotope distribution.

$\operatorname{Ae}\left(28^{*} n: 32^{*} n\right)=\operatorname{Ae}\left(28^{*} n: 32^{*} n\right)+e^{*}$ biniso; \% Even-mass convolved binomial abundance used in Figs. 2, 12, and 13.

Ao $\left(28^{*} n+1: 32^{*} n+1\right)=A o\left(28^{*} n+1: 32^{*} n+1\right)+(1-e)^{\star}$ biniso; \% Odd-mass convolved binomial abundance used in Figs. 2, 12, and 13.

end

The Gaussian expression is given in Sec. 6.2.1.

\section{Appendix C. Building simple model structures for $\mathrm{Si}_{n} \mathbf{H}_{m}$ silanions}

Fig. 8, derived from the mass spectra of Fig. 2, suggests that the silanions with maximum abundance are $\mathrm{Si}_{n} \mathrm{H}_{1.363 n}$. When the isotopic ratios were neglected in lowresolution mass spectra $[29,31]$, it appeared that the ratio was $\mathrm{Si}_{n} \mathrm{H}_{4 n / 3}$, a difference of $\sim 2 \%$. We show here that this result would imply a particularly simple rule for constructing molecular models of the most abundant silanion isomers: Each Si atom has 4 valence sites, therefore $\mathrm{Si}_{n} \mathrm{H}_{4 n / 3}$ contains a total of $4 n$ Si valence sites, of which $4 n / 3$ are occupied by $\mathrm{Si}-\mathrm{H}$ bonds. If the number of dangling bonds is negligible, then the remaining $8 n / 3 \mathrm{Si}$ valence sites are all occupied by Si-Si bonds, which implies $4 n / 3$ $\mathrm{Si}-\mathrm{Si}$ bonds, because silicon does not form multiple bonds. Hence, for $[\mathrm{H}]:[\mathrm{Si}]=4 / 3$, there are exactly equal numbers of $\mathrm{Si}-\mathrm{H}$ bonds and $\mathrm{Si}-\mathrm{Si}$ bonds in the maximum-abundance silanions $\mathrm{Si}_{n} \mathrm{H}_{4 n / 3}$. Various isomers can be conveniently fabricated using molecular bond kits by tossing a coin to choose whether each Si valence site is to be joined to an $\mathrm{H}$ or to another Si.

\section{Appendix D. Trinomial distribution for the silicon isotopes in $\mathrm{Si}_{n} \mathbf{H}_{m}^{-}$}

By analogy with the binomial distribution for the binary choice of Si or $\mathrm{H}$, the triple isotope distribution can be described by a trinomial expansion. The single and double dehydrogenation model makes the assumption of constant branching ratio $f$ for all $n$, whereas now the isotope ratios are definitive and known precisely. The binomial and 
trinomial expansions are particular cases of the general multinomial expansion. The trinomial expansion is written as:

$$
\left(p_{1}+p_{2}+p_{3}\right)^{n}=\sum_{k_{1}+k_{2}+k_{3}=n} \frac{n !}{k_{1} ! k_{2} ! k_{3} !} p_{1}^{k_{1}} p_{2}^{k_{2}} p_{3}^{k_{3}},
$$

where $\left[p_{1}, p_{2}, p_{3}\right]=[0.9221,0.0470,0.0309]$ are the isotope fractional abundances of ${ }^{28} \mathrm{Si},{ }^{29} \mathrm{Si},{ }^{30} \mathrm{Si}$ respectively, and the $n \mathrm{Si}$ atoms in $\mathrm{Si}_{n} \mathrm{H}_{m}^{-}$consist of $k_{1}{ }^{28} \mathrm{Si}, k_{2}{ }^{29} \mathrm{Si}$, and $k_{3}{ }^{30} \mathrm{Si}$ isotopes. The number of different isotopologue combinations is $\frac{(n+i-1) !}{(i-1) ! n !}=$ $(n+2)(n+1) / 2$, where $i=3$ is the number of isotopes, but for an anion containing $n$ $\mathrm{Si}$ atoms, there are only $(2 n+1)$ distinct silicon masses ranging from $28 n$ to $30 n$ amu. For $n>1$ there are therefore always more isotopologues than masses because various combinations of isotopologues have the same total mass $28 k_{1}+29 k_{2}+30 k_{3} \mathrm{amu}$. The calculated abundance of these equal-mass isotopologues must be summed to give the final $\mathrm{Si}_{n}$ mass distribution which is to be convolved with the binomial distribution of the $m \mathrm{H}$ atoms.

In Sec. 6, the single-and-double dehydrogenation model employs the binomial distribution, and the isotope distribution employs iterated convolution; these two models are then convolved to compare with the measured spectrum in Sec. 7. Alternatively, the binomial distribution could have used a double convolution; or the isotope model could have used the trinomial distribution (as shown in this appendix). Instead, we chose to use the two different methods in Sec. 6 to be more instructive, and to emphasize the different physical origins of the $\mathrm{H}$ and $\mathrm{Si}$ distributions.

\section{References}

[1] S. L. Girshick. J. Vac. Sci. Technol. A, 38:011001, 2020.

[2] L. Mangolini. J. Phys. D: Appl. Phys., 50:373003, 2017.

[3] S. J. Choi and M. J. Kushner. J. Appl. Phys., 74:853, 1993.

[4] R. Winkler, M. Capitelli, C. Gorse, and J. Wilhelm. Pl. Chem. Pl. Process., 10:419, 1990.

[5] J. Perrin, A. Lloret, G. de Rosny, and J. P. M. Schmitt. Int. J. Mass Spectrom. Ion Processes, $57: 249,1984$.

[6] M. J. Kushner. J. Appl. Phys., 63:2532, 1988.

[7] J. Perrin, C. Böhm, R. Etemadi, and A. Lloret. Plasma Sources Sci. Technol., 3:252, 1994.

[8] A. A. Fridman, L. Boufendi, T. Hbid, B. V. Potapkin, and A. Bouchoule. J. Appl. Phys., 79:1303, 1996.

[9] K.-S. Kim and M. Ikegawa. Plasma Sources Sci. Technol., 5:311, 1996.

[10] J. Perrin, O. Leroy, and M. C. Bordage. Contrib. Plasma Phys., 36:3, 1996.

[11] D.-J. Kim and K.-S. Kim. Jpn. J. Appl. Phys., 36:4989, 1997.

[12] U. R. Kortshagen, U. V. Bhandarkar, M. T. Swihart, and S. L. Girshick. Pure Appl. Chem., 71:1871, 1999.

[13] J. Perrin and Ch. Hollenstein. Chapter 24 Sources and Growth of Particles, in "Dusty Plasmas: Physics, Chemistry, and Technological Impact in Plasma Processing". New York: John Wiley \& Sons Inc., ed. A. Bouchoule, (1999).

[14] U. V. Bhandarkar, M. T. Swihart, S.L. Girshick, and U.R. Kortshagen. J. Phys. D: Appl. Phys., 33:2731, 2000.

[15] A. Gallagher, A. A. Howling, and Ch. Hollenstein. J. Appl. Phys., 91:5571, 2002.

[16] N. Gupta, W. W. Stoffels, and G. M. W. Kroesen. J. Phys. D: Appl. Phys., 36:837, 2003. 
[17] K. De Bleecker, A. Bogaerts, W. Goedheer, and R. Gijbels. IEEE Trans. Plasma Sci., 32:691, 2004.

[18] K. De Bleecker, A. Bogaerts, R. Gijbels, and W. Goedheer. Phys. Rev. E, 69:056409, 2004.

[19] A. Bogaerts, K. De Bleecker, I. Kolev, and M. Madani. Surf. Coat. Technol., 200:62, 2005.

[20] D.-J. Kim and K.-S. Kim. Ind. Eng. Chem. Res., 44:7907, 2005.

[21] Y. Watanabe. J. Phys. D: Appl. Phys., 39:R329, 2006.

[22] P. Agarwal and S. L. Girshick. Plasma Chem. Plasma Processes, 34:489, 2014.

[23] J. L. Bao, P. Seal, and D. G. Truhlar. Phys. Chem. Chem. Phys., 17:15928, 2015.

[24] J. L. Bao and D. G. Truhlar. Phys. Chem. Chem. Phys., 18:10097, 2016.

[25] A. A. Howling, J. L. Dorier, and Ch. Hollenstein. Appl. Phys. Lett., 62:1341, 1993.

[26] A. A. Howling, L. Sansonnens, J.-L. Dorier, and Ch. Hollenstein. J. Phys. D: Appl. Phys., 26:1003, 1993.

[27] A. A. Howling, L. Sansonnens, J.-L. Dorier, and Ch. Hollenstein. J. Appl. Phys., 75:1340, 1994.

[28] Ch. Hollenstein, J.-L. Dorier, J. Dutta, L. Sansonnens, and A. A. Howling. Plasma Sources Sci. Technol., 3:278, 1994.

[29] A. A. Howling, C. Courteille, J. L. Dorier, L. Sansonnens, and Ch. Hollenstein. Pure and Appl. Chem., 68:1017, 1996.

[30] C. Courteille, J.-L. Dorier, Ch. Hollenstein, L. Sansonnens, and A. A. Howling. Plasma Sources Sci. Technol., 5:210, 1996.

[31] Ch. Hollenstein, W. Schwarzenbach, A. A. Howling, C. Courteille, J.-L. Dorier, and L. Sansonnens. J. Vac. Sci. Technol. A, 14:535, 1996.

[32] Ch. Hollenstein, A. A. Howling, C. Courteille, D. Magni, S. M. Scholtz, G. M. W. Kroesen, N. Simons, W. de Zeeuw, and W. Scharzenbach. J. Phys. D: Appl. Phys., 31:74, 1998.

[33] Ch. Hollenstein, A. A. Howling, C. Courteille, J.-L. Dorier, L. Sansonnens, D. Magni, and H. Müller. Mat. Res. Soc. Symp. Proc., 507:547, 1998.

[34] W. D. Reents Jr. J. Am. Soc. Mass Spectrom., 10:918, 1999.

[35] L. Operti, R. Rabezzana, and G. A. Vaglio. Rapid Commun. Mass Spectrom., 20:2696, 2006.

[36] S. J. Peppernick, K. D. D. Gunaratne, and A. W. Castleman Jr. Int. J. Mass Spectrom., 290:65, 2010.

[37] E. Schmidt. Archaeologischen Jahrbuch, 47:246, 1932.

[38] E. S. Wallis and F. H. Adams. J. Am. Chem. Soc., 55:3838, 1933.

[39] H. Z. Sar-El. Rev. Sci. Instrum., 38:1210, 1967.

[40] J. T. Verdeyen, J. Beberman, and L. Overzet. J. Vac. Sci. Technol. A, 8:1851, 1990.

[41] R. Rabezzana. Private communication. Università di Torino, Dipartimento di Chimica, Via Giuria 710125 Torino, Italy, 2020.

[42] L. Operti, R. Rabezzana, F. Turco, and G. A. Vaglio. Rapid Commun. Mass Spectrom., 19:1963, 2005.

[43] P. Antoniotti, L. Operti, R. Rabezzana, G. A. Vaglio, and A. Guarini. Rapid Commun. Mass Spectrom., 16:185, 2002.

[44] L. Operti, R. Rabezzana, F. Turco, and G. A. Vaglio. Int. J. Mass Spectrom., 264:61, 2007.

[45] P. Haaland. J. Chem. Phys., 93:4066, 1990.

[46] P. Kofel and T. B. McMahon. Int. J. Mass Spectrom. Ion Processes, 98:1, 1990.

[47] C. H. DePuy, J. J. Grabowski, and V. M. Bierbaum. Science, 218:955, 1982.

[48] N. Mizutani, Y. Nagata, A. Kubo, and T. Hayashi. Rev. Sci. Instrum., 69:3437, 1998.

[49] M. A. Hoffmann, G. Wrigge, B. v. Issendorff, J. Müller, G. Ganteför, and H. Haberland. Eur. Phys. J. D, 16:9, 2001.

[50] A. Descoeudres, L. Sansonnens, and Ch. Hollenstein. In 29th EPS Conf. Plasma Phys. Contr. Fus., 17-21 June 2002 (Montreux, Switzerland), volume 26B, pages P-4.027, 2002.

[51] A. Descoeudres, L. Sansonnens, and Ch. Hollenstein. Plasma Sources Sci. Technol., 12:152, 2003.

[52] J. P. Booth, C. S. Corr, G. A. Curley, J. Jolly, J. Guillon, and T. Földes. Appl. Phys. Lett., $88: 151502,2006$. 
[53] P. Subramonium and M. J. Kushner. J. Vac. Sci. Technol. A, 22:534, 2004.

[54] M. A. Lieberman and A. J. Lichtenberg. Principles of Plasma Discharges and Materials Processing. John Wiley and Sons, 2nd ed., Hoboken, New Jersey, 2005.

[55] E. Illenberger and B. M. Smirnov. High Temperature, 38:829, 2000.

[56] E. Stoffels, W. W. Stoffels, and G. M. W. Kroesen. Plasma Sources Sci. Technol., 10:311, 2001.

[57] S. A. Lawton and A. V. Phelps. J. Chem. Phys., 69:1055, 1978.

[58] L. G. Christophorou, J. K. Olthoff, and M. V. V. S. Rao. J. Phys. Chem. Ref. Data, 25:134, 1996.

[59] L. G. Christophorou and J. K. Olthoff. J. Phys. Chem. Ref. Data, 28:967, 1999.

[60] L. G. Christophorou and J. K. Olthoff. J. Phys. Chem. Ref. Data, 29:267, 2000.

[61] M. Hoshino, Š. Matejčík, Y. Nunes, F. Ferreira da Silva, P. Limao-Vieira, and H. Tanaka. Int. J. Mass Spectrom., 306:51, 2011.

[62] S. K. Srivastava, E. Krishnakumar, and A. C. de A. e Souza. Int. J. Mass Spectrom. Ion Processes, 107:83, 1991.

[63] S. Pal, N. Kumar, and Anshu. Adv. Phys. Chem., 2009:309292, 2009.

[64] A. Maresca, K. Orlov, and U. Kortshagen. Phys. Rev. E, 65:056405, 2002.

[65] F. Grangeon, C. Monard, J.-L. Dorier, A. A. Howling, Ch. Hollenstein, D. Romanini, and N. Sadeghi. Plasma Sources Sci. Technol., 8:448, 1999.

[66] W. G. Xu, J. C. Yang, and W. S. Xiao. J. Phys. Chem. A, 108:11345, 2004.

[67] C. Xu, T. R. Taylor, G. R. Burton, and D. M. Neumark. J. Chem. Phys., 108:7645, 1998.

[68] J. C. Yang, X. Bai, C. P. Li, and W. G. Xu. J. Phys. Chem. A, 109:5717, 2005.

[69] N. C. Forero-Martinez, H.-L. T. Le, and H. Vach. Plasma Chem. Plasma Processes, 34:535, 2014.

[70] P. Roca i Cabarrocas, P. Gay, and A. Hadjadj. J. Vac. Sci. Technol. A, 14:655, 1996.

[71] P. Roca i Cabarrocas. J. Non-Cryst. Solids, 266:31, 2000.

[72] U. Boesl and W. J. Knott. Mass Spectrom. Rev., 17:275, 1998.

[73] L. A. Pinnaduwage, M. Z. Martin, and L. G. Christophorou. Appl. Phys. Lett., 65:2571, 1994.

[74] L. A. Pinnaduwage and P. G. Datskos. J. Appl. Phys., 81:7715, 1997.

[75] C. H. DePuy. J. Org. Chem., 67:2393, 2002.

[76] MATLAB. R2018b. The MathWorks Inc., Natick, Massachusetts. 